\title{
Fairness-Aware Energy-Efficient Resource Allocation for AF Cooperative OFDMA Networks
}

\author{
Ebrahim Bedeer, Member, IEEE, Abdulaziz Alorainy, Student Member, IEEE, \\ Md. Jahangir Hossain, Member, IEEE, Osama Amin, Senior Member, IEEE, \\ and Mohamed-Slim Alouini, Fellow, IEEE
}

\begin{abstract}
In this paper, we adopt an energy-efficiency (EE) metric, named worst-EE, that is suitable for $\mathrm{EE}$ fairness optimization in the uplink transmission of amplify-and-forward (AF) cooperative orthogonal frequency division multiple access (OFDMA) networks. More specifically, we assign subcarriers and allocate powers for mobile and relay stations in order to maximize the worst-EE, i.e., to maximize the $\mathrm{EE}$ of the mobile station (MS) with the lowest EE value, subject to MSs transmit power, relay station (RS) transmit power, and MSs quality-ofservice (QoS) constraints. The formulated primal $\max -\min$ optimization problem is nonconvex fractional mixed integer nonlinear program that is NP-hard to solve. We provide a novel optimization framework that studies the structure of the primal problem and prove that the dual min - max optimization problem attains the same optimal solution of the primal problem. Additionally, we propose a modified Dinkelbach algorithm, named dual Dinkelbach, to achieve the optimal solution of the dual problem in a polynomial time complexity. We further exploit the structure of the obtained optimal solution and develop a low complexity suboptimal heuristic. Numerical results show the effectiveness of the proposed algorithm to improve the network performance in terms of fairness between MSs, worst-EE, and average network transmission rate when compared to traditional schemes that maximize the EE of the whole network. Presented results also show that the suboptimal heuristic balances the achieved performance and the computational complexity.
\end{abstract}

Index Terms-Amplify-and-forward (AF), cooperative communications, energy-efficiency, green communications, OFDMA, uplink transmission.

\section{INTRODUCTION}

In recent years, energy-efficient communications have received a lot of attention from both industry and academia due to the hike in energy consumption costs, ecological, and environmental reasons [1]-[3]. Several wireless standards have

Manuscript received March 15, 2015; revised July 15, 2015; accepted September 4, 2015. This work has been supported in part by Natural Science and Engineering Research Council of Canada (NSERC) through Discovery program. The work of M. -S. Alouini has been supported by the Qatar National Research Fund (a member of Qatar Foundation) under NPRP Grant NPRP 5-250-2-087. The statements made herein are solely the responsibility of the authors.

Ebrahim Bedeer, Abdulaziz Alorainy, and Md. Jahangir Hossain are with the School of Engineering, the University of British Columbia (UBC), Kelowna, BC, V1V 1V7, Canada (e-mail: ebrahim.bedeer-mohamed@ubc.ca abdulaziz.alorainy@alumni.ubc.ca, and jahangir.hossain@ubc.ca).

Abdulaziz Alorainy is also with King Abdulaziz City for Science and Technology (KACST), Riyadh, Saudi Arabia (e-mail: aalorainy@kacst.edu.sa).

Osama Amin and Mohamed-Slim Alouini are with the Computer, Electrical and Mathematical Sciences and Engineering (CEMSE) Division, King Abdullah University of Science and Technology (KAUST), Thuwal, Makkah Province, Saudi Arabia (e-mail: osama.amin@kaust.edu.sa and slim.alouini@kaust.edu.sa). introduced relay stations (RSs) in order to improve the network performance [4]. With appropriate selection/deployment of RSs in the network, the energy-efficiency (EE) of communication systems can be improved [5], as the path loss between mobile stations (MSs) and the base station (BS) can be reduced. As such, the same quality of transmission can be maintained at a reduced transmit power, or the quality can be further improved for the same transmit power level. One of the simplest and most popular relaying techniques is amplify-andforward (AF) [6], where the source broadcasts its information to the RS in the first time slot and in the second time slot the RS linearly amplifies the received signal from the source and forwards it to the destination. Orthogonal frequency division multiple access (OFDMA) is adopted in many contemporary wireless standards [7] due to its inherent advantages and flexibilities [8]. Several works in the literature combined both OFDMA and relaying in order to get the benefits of both techniques (see, e.g., [9]-[12] and references therein); however, such classical designs, which tend to maximize transmission rate, capacity and coverage, potentially lead to solutions that degrade the energy efficiency performance.

Recently, a flourish of works has been done for maximizing the EE of cooperative OFDMA systems [13]-[18]. More specifically, Chen et al. [13] proposed a suboptimal algorithm to maximize the $\mathrm{EE}$ of $\mathrm{AF}$ orthogonal frequency division multiplexing (OFDM)-based system. The proposed algorithm considered a simplified scenario of a single source node communicating with a single destination node through a single relay. In [14], Sun et al. considered an OFDMA relay system where the communication between two nodes is achieved with the assistance of an AF relay node. The authors proposed to optimize the number of active subcarriers and the number of transmitted bits on each subcarrier in order to minimize the overall power consumption of the network, and hence, improve the EE. Devarajan et al. in [15] proposed a multi-objective optimization approach to simultaneously maximize the achievable transmission rate and minimize the transmit power in order to improve the EE of a cooperative cellular system. This is achieved by linearly combining the transmission rate and transmit power using corresponding weighting coefficients into a single objective. However, the circuitry processing power was not considered and the choice of the weighting coefficients that maximize the EE was not discussed. For an OFDMA relay network, Ho and Huang in [16] considered a RS to assist the transmission of multiple MSs to a single BS. The authors optimized the subcarrier allocation between 
users and the power allocated to each subcarrier in order to maximize the EE of the whole network. In [17], Cheung et al. studied the downlink of an OFDMA cellular system where the communication between BS and MSs is achieved through a single RS in each sector. An optimization problem, considering AF relaying, was formulated to optimally allocate the power and subcarriers in order to maximize the average EE of the network. Loodaricheh et al. [18] extended the model in [17] to accommodate relay selection to further improve the EE performance.

The goal of any resource allocation algorithm is to better utilize the network resources, given a specific transmission scenario, while providing a satisfactory level of performance for each user (in terms of the EE of each MS in our case). That said, a resource allocation algorithm designed for downlink transmission scenarios may not be suitable for uplink transmission scenarios. The aforementioned works maximize the EE of the whole network, named global-EE, that is defined as the ratio between the total achieved network throughput/transmission rate and the total transmit signal and circuitry processing power. Although such EE metric, i.e., global-EE, can improve the overall EE of the whole network, there is no guarantee on the achieved individual EE of MSs. For example, global-EE maximization may favor MSs that are geographically close to the $\mathrm{BS}$ or RS compared to MSs at the cell edge that usually starve. For uplink scenarios, MSs want to maximize their own benefits, e.g., EE in our case, and hence, it is crucial to improve the EE of individual MSs. This is especially important given that each MS is equipped with its own battery with limited power supply. That said, adopting the global-EE metric may not be appropriate for uplink transmission scenarios as it can lead to unfairness in terms of EE of the MSs.

In this work, we consider the uplink transmission scenario of an OFDMA cooperative cellular system and propose to allocate the power and subcarriers of mobile and relay stations in order to maximize the worst-EE, i.e., to maximize the EE of the MS with the lowest EE value. It is worthy to mention that similar metric was proposed for a simpler system model (i.e., non-cooperative with no RSs) [19]; however, the optimal solution was obtained only through an exhaustive search. The main contributions of this paper are summarized as follows:

- We consider an EE fairness optimization metric that is suitable for the uplink resource allocation problem for AF cooperative OFDMA networks. More specifically, the subcarrier and power allocation problem of mobile and relay stations is formulated as a primal $\max -\min$ optimization problem that maximizes the worst-EE subject to maximum MSs transmit powers, maximum RS transmit power, and minimum MSs supported transmission rate constraints. The problem is challenging given the diverse transmission rate and power constraints of the MSs and $\mathrm{RS}$; in contrast to works for the downlink in the literature [16]-[18] that assume single transmit power constraint of the whole network (i.e., BS and RS).

- The formulated primal max - min optimization problem is nonconvex fractional mixed integer nonlinear program that is NP-hard to solve. To facilitate obtaining the solution, the integer constraints are relaxed; however, the resultant optimization problem is still computationally complex to find the optimal solution. To reduce the computational complexity, we study the structure of the primal max - min problem and find the equivalent dual min - max optimization problem, where we prove that both problems attain the same optimal solution. Then, the optimal allocation of subcarriers and powers of mobile and relay stations of the dual min - max problem is derived based on concepts of fractional programming and dual decomposition.

- We propose a modified Dinkelbach algorithm, named dual Dinkelbach, to achieve the optimal solution of the dual min - max problem with polynomial time complexity. By exploring the inherent structure of the optimal solution, we also develop a low complexity suboptimal heuristic with comparable performance to the optimal solution.

- Extensive simulation results are provided to show the merits of the worst-EE metric optimization for uplink scenarios. Results show the benefits of the proposed optimal scheme to improve fairness (in terms of EE) between different MSs, to improve worst-EE, as well as to increase the average transmission rate of the network when compared to schemes in the literature that maximize the global-EE. Additionally, results show that comparable performance can be achieved using the suboptimal heuristic at reduced computational complexity.

The remainder of this paper is organized as follows. While Section II introduces the system model, Section III formulates and analyzes the primal $\max -\min$ and dual $\min -\max$ problems. Section IV solves the dual min - max problem and proposes a modified Dinkelbach algorithm, named dual Dinkelbach, to achieve the optimal solution. In this Section, we also propose a low complexity suboptimal heuristic. Simulation results are presented in Section V, while conclusions are drawn in Section VI.

Throughout the paper we use bold-faced upper case letters, e.g., $\mathbf{X}$, to denote matrices and bold-faced lower case letters, e.g., $\mathbf{x}$, for vectors. [.] $]^{\mathrm{T}}$ denotes the transpose operation, $[x]^{+}$ represents $\max (0, x), \mathbf{I}$ is the identity matrix, $x^{*}$ is the optimal value of $x$, and calligraphic letters, i.e., $\mathcal{X}$, are used to denote sets.

\section{SySTEM MODEL}

Consider a cellular OFDMA network, where the cell is divided into a number of sectors with each sector is served by a RS. Within each sector, we assume the existence of $K$ uniformly distributed MSs communicating with a BS through the assistance of a RS, as shown in Fig. 1. We focus on one sector for the sake of mathematical tractability, however, the model can be readily extended to include all sectors within a cell. Additionally, we assume the existence of $N$ orthogonal subcarriers each of bandwidth $B$. We assume that MSs communicate with the BS through the direct link as well as through the RS in order to exploit diversity combining.

AF cooperation is assumed where broadcasting from the MS $k \in\{1, \ldots, K\}$ to the RS and to the BS is achieved in the 


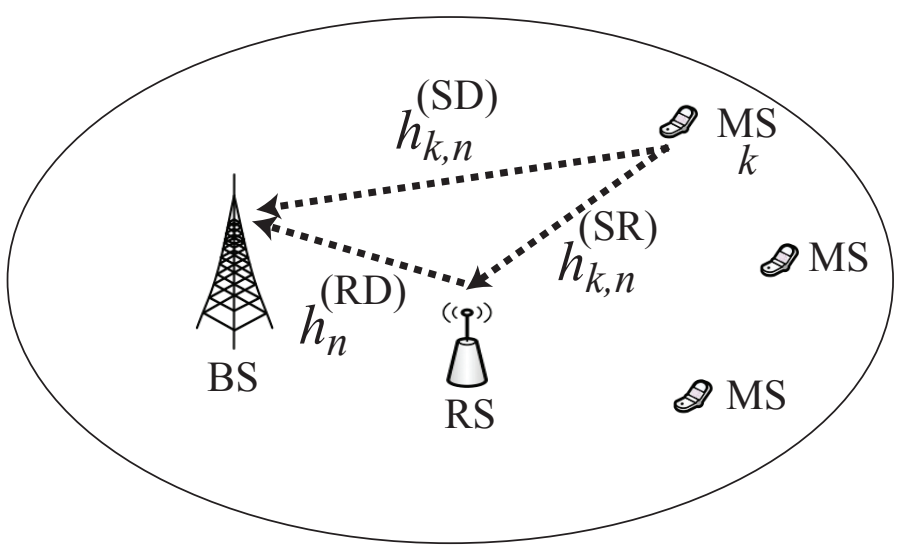

Fig. 1: System model of a cellular OFDMA cooperative network showing the tagged relay.

first time slot and transmission from the RS to the BS is done in the second time slot. The received signals at the RS and $\mathrm{BS}, y_{k, n}^{(\mathrm{R}, 1)}$ and $y_{k, n}^{(\mathrm{D}, 1)}$, respectively, from MS $k$ on subcarrier $n \in\{1, \ldots, N\}$ in the first time slot are given by [20]

$$
\begin{aligned}
& y_{k, n}^{(\mathrm{R}, 1)}=\sqrt{p_{k, n}^{(\mathrm{S})}} h_{k, n}^{(\mathrm{SR})} x_{k, n}+n_{k, n}^{(\mathrm{R}, 1)}, \\
& y_{k, n}^{(\mathrm{D}, 1)}=\sqrt{p_{k, n}^{(\mathrm{S})}} h_{k, n}^{(\mathrm{SD})} x_{k, n}+n_{k, n}^{(\mathrm{D}, 1)},
\end{aligned}
$$

where $p_{k, n}^{(\mathrm{S})}$ and $x_{k, n}$ are the transmit power and the transmit symbol of MS $k$ on subcarrier $n$, respectively, $h_{k, n}^{(\mathrm{SR})}$ and $h_{k, n}^{(\mathrm{SD})}$ are the channel coefficients between MS $k$ and both the RS and BS on subcarrier $n$, respectively, and $n_{k, n}^{(\mathrm{R}, 1)}$ and $n_{k, n}^{(\mathrm{D}, 1)}$ are additive while Gaussian noise (AWGN) with zero mean and variance $\sigma_{z}^{2}$ at the RS and BS in the first time slot, respectively. In the second time slot, the RS amplifies and forwards the received signal at the same subcarrier ${ }^{1}$. The received signal $y_{k, n}^{(\mathrm{D}, 2)}$ of MS $k$ on subcarrier $n$ in the second time slot at the $\mathrm{BS}$ is given as [20]

$$
y_{k, n}^{(\mathrm{D}, 2)}=\Omega_{k, n} \sqrt{p_{k, n}^{(\mathrm{R})}} h_{n}^{(\mathrm{RD})} y_{k, n}^{(\mathrm{R}, 1)}+n_{k, n}^{(\mathrm{D}, 2)},
$$

where $p_{k, n}^{(\mathrm{R})}$ is the power of $\mathrm{RS}$ on subcarrier $n$ to support the transmission of MS $k, h_{n}^{(\mathrm{RD})}$ is the channel coefficients between the RS and BS on subcarrier $n, n_{k, n}^{(\mathrm{D}, 2)}$ is the AWGN with zero mean and variance $\sigma_{z}^{2}$ at the BS in the second time slot, and $\Omega_{k, n}=\left(p_{k, n}^{(\mathrm{S})} /\left(p_{k, n}^{(\mathrm{S})}\left|h_{k, n}^{(\mathrm{SR})}\right|^{2}+\sigma_{z}^{2}\right)\right)^{1 / 2}$ [6] is the normalization factor at the RS associated with MS $k$ on subcarrier $n$.

At the end of the second time slot, the BS combines the direct link signal received from MS $k$ and the one received through the RS using a maximal ratio combining [6]. Thus, the signal-to-noise ratio (SNR) of the link between MS $k$ and $\mathrm{BS}$ on subcarrier $n$ is given as [21]

$$
\Gamma_{k, n}^{(\mathrm{CL})}=\Gamma_{k, n}^{(\mathrm{DL})}+\Gamma_{k, n}^{(\mathrm{TH})},
$$

where $\Gamma_{k, n}^{(\mathrm{DL})}=p_{k, n}^{(\mathrm{S})} \gamma_{k, n}^{(\mathrm{SD})}$ is the SNR of the direct link between MS $k$ and the BS on subcarrier $n$ with $\gamma_{k, n}^{(\mathrm{SD})}=$

\footnotetext{
${ }^{1}$ It was shown in [13], [18] that subcarrier pairing, i.e., using different subcarriers on the links between MS-RS and RS-BS, provides marginal improvements in performance at the expense of huge computational complexity.
}

$\frac{\left|h_{k, n}^{(\mathrm{SD})}\right|^{2} G_{k}^{(\mathrm{SD})}}{\sigma_{z}^{2}}$ is the channel gain-to-noise ratio (CNR) of MS $k$ on subcarrier $n$ to the BS and $G_{k}^{(\mathrm{SD})}$ is the path loss between the $k$ th MS and the BS. $\Gamma_{k, n}^{(\mathrm{TH})}$ is the two-hop SNR of the link between the MS $k$ on subcarrier $n$ to the BS through the RS and it is given as [20]

$$
\begin{aligned}
\Gamma_{k, n}^{(\mathrm{TH})} & =\frac{p_{k, n}^{(\mathrm{S})} \gamma_{k, n}^{(\mathrm{SR})} p_{k, n}^{(\mathrm{R})} \gamma_{n}^{(\mathrm{RD})}}{1+p_{k, n}^{(\mathrm{S})} \gamma_{k, n}^{(\mathrm{SR})}+p_{k, n}^{(\mathrm{R})} \gamma_{n}^{(\mathrm{RD})}} \\
& \simeq \frac{p_{k, n}^{(\mathrm{S})} \gamma_{k, n}^{(\mathrm{SR})} p_{k, n}^{(\mathrm{R})} \gamma_{n}^{(\mathrm{RD})}}{p_{k, n}^{(\mathrm{S})} \gamma_{k, n}^{(\mathrm{SR})}+p_{k, n}^{(\mathrm{R})} \gamma_{n}^{(\mathrm{RD})}},
\end{aligned}
$$

where $\gamma_{k, n}^{(\mathrm{SR})}=\frac{\left|h_{k, n}^{(\mathrm{SR})}\right|^{2} G_{k}^{(\mathrm{SR})}}{\sigma_{z}^{2}}$ and $\gamma_{n}^{(\mathrm{RD})}=\frac{\left|h_{n}^{(\mathrm{RD})}\right|^{2} G^{(\mathrm{RD})}}{\sigma_{z}^{2}}$ are the CNR from MS $k$ to the RS and from the RS to the BS, respectively, on subcarrier $n$ and $G_{k}^{(\mathrm{SR})}$ and $G^{(\mathrm{RD})}$ are the path losses between MS $k$ and the RS and between the RS and BS, respectively. The approximation of $\Gamma_{k, n}^{(\mathrm{TH})}$ in (5) is tight at higher SNR values [20] and it is also valid at low SNR values [22].

The transmission rate of MS $k, r_{k}$, is expressed as [6]

$$
r_{k}\left(p_{k, n}^{(\mathrm{S})}, p_{k, n}^{(\mathrm{R})}, \phi_{k, n}\right)=\frac{B}{2} \sum_{n=1}^{N} \phi_{k, n} \log _{2}\left(1+\frac{\Gamma_{k, n}^{(\mathrm{CL})}}{\Gamma}\right),
$$

where $\Gamma$ is the SNR gap that is a function of the system bit error rate, and it is defined as the difference between the SNR required to achieve the channel capacity and the actual SNR required by a certain modulation and coding schemes, $\phi_{k, n}$ is an integer variable if 1 then subcarrier $n$ is allocated to MS $k$; otherwise it is 0 , and the factor $\frac{1}{2}$ to account that the transmission occurs over two time slots. The total power consumption of MS $k, p_{k}$, can be formulated as

$$
p_{k}\left(p_{k, n}^{(\mathrm{S})}, \phi_{k, n}\right)=\frac{1}{2}\left(p_{k, \mathrm{c}}+\frac{1}{\xi_{k}^{(\mathrm{S})}} \sum_{n=1}^{N} \phi_{k, n} p_{k, n}^{(\mathrm{S})}\right),
$$

where $p_{k, \mathrm{c}}$ is the total circuitry power consumption required to deliver the information of MS $k$ and $\xi_{k}^{(\mathrm{S})}$ is the power amplifier efficiency of MS $k$. Finally, the EE of MS $k, \eta_{k}$, that is defined as ratio of the transmission rate of MS $k$ divided by its total power consumption is formally expressed as

$$
\begin{aligned}
\eta_{k}\left(p_{k, n}^{(\mathrm{S})}, p_{k, n}^{(\mathrm{R})}, \phi_{k, n}\right)=\frac{r_{k}\left(p_{k, n}^{(\mathrm{S})}, p_{k, n}^{(\mathrm{R})}, \phi_{k, n}\right)}{p_{k}\left(p_{k, n}^{(\mathrm{S})}, \phi_{k, n}\right)} \\
=\frac{B \sum_{n=1}^{N} \phi_{k, n} \log _{2}\left(1+\left(\Gamma_{k, n}^{(\mathrm{DL})}+\Gamma_{k, n}^{(\mathrm{TH})}\right) / \Gamma\right)}{p_{k, \mathrm{c}}+\frac{1}{\xi_{k}^{(\mathrm{S})}} \sum_{n=1}^{N} \phi_{k, n} p_{k, n}^{(\mathrm{S})}}
\end{aligned}
$$

\section{Primal And Dual Optimization Problems: FORMULATION \& ANALYSIS}

In this section, we formulate the subcarriers and powers allocation problem of mobile and relay stations of AF cooperative communication for the uplink transmission scenario of OFDMA networks. A primal max-min optimization problem is formulated that maximizes the worst-EE subject to maximum transmit power per each MS, maximum transmit power of the RS, and minimum supported transmission rate per each MS. We analyze the structure of the primal max - min 


$$
\eta_{\text {global }-\mathrm{EE}}=\max _{\mathbf{P}^{(\mathrm{S})}, \mathbf{P}^{(\mathrm{R})}, \mathbf{\Phi}} \frac{\sum_{k=1}^{K} w_{k} r_{k}\left(p_{k, n}^{(\mathrm{S})}, p_{k, n}^{(\mathrm{R})}, \phi_{k, n}\right)}{\frac{1}{2} \sum_{k=1}^{K} p_{k, \mathrm{c}}+\frac{1}{2 \xi_{k}^{(\mathrm{S})}} \sum_{n=1}^{N} \phi_{k, n} p_{k, n}^{(\mathrm{S})}+\frac{1}{2 \xi_{k}^{(\mathrm{R})}} \sum_{n=1}^{N} \phi_{k, n} p_{k, n}^{(\mathrm{R})}},
$$

and show that the equivalent dual $\min -\max$ attains the same optimal solution.

\section{A. Primal max - min Problem}

The global-EE metric used for the downlink scenario [16][18] that maximizes the average EE of the whole network can be expressed as in (9) on the top of this page, where $\mathbf{P}^{(\mathrm{S})}$, $\mathbf{P}^{(\mathrm{R})}$, and $\boldsymbol{\Phi}$ are the $K \times N$ matrices of MSs power allocation, RS power allocation, and subcarrier assignment, respectively, and $w_{k}\left(\sum_{k=1}^{K} w_{k}=1\right)$ is a weighting coefficient that is used to reflect fairness between MSs. As can be seen in (9), $w_{k}$ is not directly related to the EE of MS $k, \eta_{k}$; however, it is related to its transmission rate, $r_{k}$. Hence, it may not be accurate to claim that $w_{k}$ reflects fairness between MSs in terms of EE. A possible way to address the EE fairness problem is to maximize the worst-EE, i.e., the EE of MS with the lowest EE value. The approach is beneficial for the uplink scenario where it is crucial to improve individual EE of each MS. That said, the goal of this paper is to improve fairness between MSs in terms of EE for an $\mathrm{AF}^{2}$ uplink scenario. Thus, we formulate the following $\max -\min$ optimization problem

$$
\begin{aligned}
& \eta_{\mathrm{worst}-\mathrm{EE}}=\max _{\mathbf{P}^{(\mathrm{S})}, \mathbf{P}^{(\mathrm{R})}, \mathbf{\Phi}} \min _{1 \leq k \leq K} \eta_{k}\left(p_{k, n}^{(\mathrm{S})}, p_{k, n}^{(\mathrm{R})}, \phi_{k, n}\right) \\
&=\max _{\mathbf{P}^{(\mathrm{S})}, \mathbf{P}^{(\mathrm{R})}, \mathbf{\Phi}} \min _{1 \leq k \leq K} \frac{r_{k}\left(p_{k, n}^{(\mathrm{S})}, p_{k, n}^{(\mathrm{R})}, \phi_{k, n}\right)}{p_{k}\left(p_{k, n}^{(\mathrm{S})}, \phi_{k, n}\right)} \\
& \mathrm{C} 1: \sum_{k=1}^{K} \sum_{n=1}^{N} \phi_{k, n} p_{k, n}^{(\mathrm{R})} \leq p_{\max }^{(\mathrm{R})} \\
& \mathrm{C} 2: \sum_{n=1}^{N} \phi_{k, n} p_{k, n}^{(\mathrm{S})} \leq p_{k, \max }^{(\mathrm{S})} \forall k \\
& \text { s.t. } \quad \mathrm{C} 3: r_{k}\left(p_{k, n}^{(\mathrm{S})}, p_{k, n}^{(\mathrm{R})}, \phi_{k, n}\right) \geq r_{k, \min }^{(\mathrm{S})} \quad \forall k \\
& \\
& \mathrm{C} 4: p_{k, n}^{(\mathrm{S})} \geq 0, p_{k, n}^{(\mathrm{R})} \geq 0 \quad \forall k, n \\
& \mathrm{C} 5: \phi_{k, n} \in\{0,1\} \quad \forall k, n \\
& \mathrm{C} 6: \sum_{k=1}^{K} \phi_{k, n}=1 \quad \forall n,
\end{aligned}
$$

The constraint $\mathrm{C} 1$ limits the power of the RS to forward the data of all the MSs on all the subcarriers to a maximum value $p_{\max }^{(\mathrm{R})}$, the constraint $\mathrm{C} 2$ limits the transmit power of MS $k$ to a certain power budget $p_{k, \max }^{(\mathrm{S})}$, the constraint C3 is to guarantee a minimum supported transmission rate $r_{k, \text { min }}^{(\mathrm{S})}$ for MS $k$, the constraint $\mathrm{C} 4$ is to guarantee positive power allocation to MS $k$ and the $\mathrm{RS}$ on subcarrier $n$, the constraint

\footnotetext{
${ }^{2}$ In uplink decode-and-forward (DF) relaying, the broadcast from MS $k \in$ $\{1, \ldots, K\}$ to RS and BS is achieved in the first time slot. While, in the second time slot, the RS transmits an encoded version of its received signal to the BS [6]. In order to correctly decode the transmit symbols at the RS, the MS $k$ transmission rate on subcarrier $n$ should not be higher than [6] $r_{k, n}^{\mathrm{RS}}=\log _{2}\left(1+\left(p_{k, n}^{(\mathrm{S})} \gamma_{k, n}^{(\mathrm{SR})}\right) / \Gamma\right)$. On the other hand, to ensure reliable decoding of transmit symbols at the BS, the MS $k$ transmission rate should not be higher than $r_{k, n}^{\mathrm{BS}}=\log _{2}\left(1+\left(p_{k, n}^{(\mathrm{S})} \gamma_{k, n}^{(\mathrm{SD})}+p_{k, n}^{(\mathrm{R})} \gamma_{k, n}^{(\mathrm{RD})}\right) / \Gamma\right)$. That said, the transmission rate of MS $k$ on subcarrier $n$ for DF relying is expressed as $r_{k, n}=\frac{B}{2} \min \left\{r_{k, n}^{\mathrm{RS}}, r_{k, n}^{\mathrm{BS}}\right\}$, and the transmission rate of MS $k$ can be written as $r_{k}=\frac{B}{2} \sum_{n=1}^{N} \phi_{k, n} r_{k, n}$. The worst-EE maximization problem for DF relaying can be formulated similar to (10); however, the analysis and the solution will not discussed in this paper due to space limitations.
}

C5 is to reflect the integer nature of the variable $\phi_{k, n}$, and the constraint $\mathrm{C} 6$ reflects that subcarrier $n$ can be assigned to a single MS at most. The problem in (10) is a mixed-integer nonlinear programming (MINP) problem that is NP-hard to solve with the complexity increases at least exponentially with the problem size $K N$ [23]. A common way to tackle integer variables is through time-sharing principle [24], i.e., by relaxing the integer variables into continuous ones to allow MSs to share subcarriers. Obviously, the optimal solution of the relaxed continuous optimization problem is always an upper bound of the original MINP problem as all the feasible solutions to the original problem fall into the solution space of the relaxed continuous problem. It was shown in [24], [25] that solving the relaxed continuous problem provides solutions that is close to the solutions of the original MINP problem given that the number of subcarriers tends to infinity. Additionally, [17], [26] showed empirically that the gap can be tight even for small number of subcarriers. That said, the integer variable $\phi_{k, n} \in\{0,1\}$ is relaxed to a continuous one $\theta_{k, n} \in[0,1]$. Additionally, we introduce the following auxiliary power variables, $\widetilde{p}_{k, n}^{(\mathrm{S})}=p_{k, n}^{(\mathrm{S})} \theta_{k, n}$ and $\widetilde{p}_{k, n}^{(\mathrm{R})}=p_{k, n}^{(\mathrm{R})} \theta_{k, n}$, to facilitate obtaining the solution and to characterize the actual transmit power of MS $k$ and the RS, respectivelly, on subcarrier $n$. The auxiliary relaxed optimization problem is written as

$$
\begin{aligned}
\mathfrak{P}: & \max _{\widetilde{\mathbf{P}}^{(\mathrm{S})}, \widetilde{\mathbf{P}}^{(\mathrm{R})}, \Theta} \min _{1 \leq k \leq K} \frac{\widetilde{r}_{k}\left(\widetilde{p}_{k, n}^{(\mathrm{S})}, \widetilde{p}_{k, n}^{(\mathrm{R})}, \theta_{k, n}\right)}{\widetilde{p}_{k}\left(\widetilde{p}_{k, n}^{(\mathrm{S})}, \theta_{k, n}\right)} \\
& \widetilde{\mathrm{C} 1}: \sum_{k=1}^{K} \sum_{n=1}^{N} \widetilde{p}_{k, n}^{(\mathrm{R})} \leq p_{\max }^{(\mathrm{R})} \\
& \widetilde{\mathrm{C} 2}: \sum_{n=1}^{N} \widetilde{p}_{k, n}^{(\mathrm{S})} \leq p_{k, \max }^{(\mathrm{S})} \quad \forall k \\
& \widetilde{\mathrm{C} 3}: \widetilde{r}_{k}\left(\widetilde{p}_{k, n}^{(\mathrm{S})}, \widetilde{p}_{k, n}^{(\mathrm{R})}, \theta_{k, n}\right) \geq r_{k, \min }^{(\mathrm{S})} \quad \forall k \\
\text { s.t. } & \widetilde{\mathrm{C}} 4: \widetilde{p}_{k, n}^{(\mathrm{S})} \geq 0, \widetilde{p}_{k, n}^{(\mathrm{R})} \geq 0 \quad \forall k, n \\
& \widetilde{\mathrm{C} 5}: \theta_{k, n} \in[0,1] \quad \forall k, n \\
& \widetilde{\mathrm{C} 6}: \sum_{k=1}^{K} \theta_{k, n}=1 \quad \forall n,
\end{aligned}
$$

where $\widetilde{\mathbf{P}}^{(\mathrm{S})}, \widetilde{\mathbf{P}}^{(\mathrm{R})}$, and $\Theta$ are the $K \times N$ matrices of auxiliary MSs power allocation, auxiliary RS power allocation, and subcarrier assignment, respectively, and

$$
\begin{aligned}
& \widetilde{r}_{k}\left(\widetilde{p}_{k, n}^{(\mathrm{S})}, \widetilde{p}_{k, n}^{(\mathrm{R})}, \theta_{k, n}\right)=\frac{B}{2} \sum_{n=1}^{N} \theta_{k, n} \\
& . \log _{2}\left(1+\frac{1}{\Gamma \theta_{k, n}}\left(\widetilde{p}_{k, n}^{(\mathrm{S})} \gamma_{k, n}^{(\mathrm{SD})}+\frac{\widetilde{p}_{k, n}^{(\mathrm{S})} \gamma_{k, n}^{(\mathrm{SR})} \widetilde{p}_{k, n}^{(\mathrm{R})} \gamma_{n}^{(\mathrm{RD})}}{\widetilde{p}_{k, n}^{(\mathrm{S})} \gamma_{k, n}^{(\mathrm{SR})}+\widetilde{p}_{k, n}^{(\mathrm{R})} \gamma_{n}^{(\mathrm{RD})}}\right)\right),
\end{aligned}
$$$$
\widetilde{p}_{k}\left(\widetilde{p}_{k, n}^{(\mathrm{S})}, \theta_{k, n}\right)=\frac{1}{2}\left(p_{k, \mathrm{c}}^{(\mathrm{S})}+\frac{1}{\xi_{k}^{(\mathrm{S})}} \sum_{n=1}^{N} \widetilde{p}_{k, n}^{(\mathrm{S})}\right) .
$$

Let us denote the problem in (11) as the generalized primal $\max -\min$ fractional problem $\mathfrak{P}$. We show in Appendix A that the objective function of the primal problem $\mathfrak{P}$ is 
quasi-concave in $\widetilde{p}_{k, n}^{(\mathrm{S})}, \widetilde{p}_{k, n}^{(\mathrm{R})}$, and $\theta_{k, n}$, as $\widetilde{r}_{k}\left(\widetilde{p}_{k, n}^{(\mathrm{S})}, \widetilde{p}_{k, n}^{(\mathrm{R})}, \theta_{k, n}\right)$ is concave and $\widetilde{p}_{k}\left(\widetilde{p}_{k, n}^{(\mathrm{S})}, \theta_{k, n}\right)$ is affine. Such property allows us to adopt the primal Dinkelbach algorithm [27] to solve a sequence of parameterized concave problems. The problem $\mathfrak{P}$ can be rewritten in the parametric form as

$$
\begin{gathered}
\max _{\widetilde{\mathbf{P}}^{(\mathrm{S})}, \widetilde{\mathbf{P}}^{(\mathrm{R})}, \Theta} \min _{1 \leq k \leq K} \widetilde{r}_{k}\left(\widetilde{p}_{k, n}^{(\mathrm{S})}, \widetilde{p}_{k, n}^{(\mathrm{R})}, \theta_{k, n}\right)-q \widetilde{p}_{k}\left(\widetilde{p}_{k, n}^{(\mathrm{S})}, \theta_{k, n}\right) \\
\text { s.t. } \widetilde{\mathrm{C} 1}, \widetilde{\mathrm{C}} 2, \widetilde{\mathrm{C}} 3, \widetilde{\mathrm{C}} 4, \widetilde{\mathrm{C}} 5, \widetilde{\mathrm{C}} 6,
\end{gathered}
$$

where $q$ is a non-negative parameter. The parametric problem in (14) can be solved at a certain value of $q$ using convex optimization techniques [28], then the value of $q$ is updated ${ }^{3}$ until convergence to the optimal $q^{*}$ defined in (16). The solution of the primal problem at a certain value of $q$ is computationally complex. In the following, we find sufficient conditions under which the primal max $-\min$ and dual min - max problems attain the same solution. This enables us to solve the dual min - max problem in Section IV.

In order to analyze of the primal max - min optimization problem $\mathfrak{P}$, let us define the following single-ratio fraction optimization problem as follows

$$
q^{*}\left(\widetilde{p}_{k, n}^{(\mathrm{S})}, \widetilde{p}_{k, n}^{(\mathrm{R})}, \theta_{k, n}\right)=\min _{1 \leq k \leq K} \frac{\widetilde{r}_{k}\left(\widetilde{p}_{k, n}^{(\mathrm{S})}, \widetilde{p}_{k, n}^{(\mathrm{R})}, \theta_{k, n}\right)}{\widetilde{p}_{k}\left(\widetilde{p}_{k, n}^{(\mathrm{S})}, \theta_{k, n}\right)} .
$$

Accordingly, the primal max - min optimization problem $\mathfrak{P}$ can be rewritten as

$$
q^{*}=\max _{\left(\widetilde{\mathbf{P}}^{(\mathrm{S})}, \widetilde{\mathbf{P}}^{(\mathrm{R})}, \Theta\right) \in \mathcal{A}} q^{*}\left(\widetilde{p}_{k, n}^{(\mathrm{S})}, \widetilde{p}_{k, n}^{(\mathrm{R})}, \theta_{k, n}\right),
$$

where $\mathcal{A}$ is the nonempty closed set defined by the constraints $\widetilde{\mathrm{C}} 1, \widetilde{\mathrm{C}} 2, \widetilde{\mathrm{C}} 3, \widetilde{\mathrm{C}} 4, \widetilde{\mathrm{C} 5}$, and $\widetilde{\mathrm{C}} 6$. Additionally, let us define the following function

$$
\begin{aligned}
& f_{k}\left(q, \widetilde{p}_{k, n}^{(\mathrm{S})}, \widetilde{p}_{k, n}^{(\mathrm{R})}, \theta_{k, n}\right) \\
& \quad=\widetilde{r}_{k}\left(\widetilde{p}_{k, n}^{(\mathrm{S})}, \widetilde{p}_{k, n}^{(\mathrm{R})}, \theta_{k, n}\right)-q \widetilde{p}_{k}\left(\widetilde{p}_{k, n}^{(\mathrm{S})}, \theta_{k, n}\right),
\end{aligned}
$$

and consider for every $\left(q, \widetilde{p}_{k, n}^{(\mathrm{S})}, \widetilde{p}_{k, n}^{(\mathrm{R})}, \theta_{k, n}\right)$ the following parametric optimization problems

$$
\begin{array}{r}
f^{(1)}\left(q, \widetilde{p}_{k, n}^{(\mathrm{S})}, \widetilde{p}_{k, n}^{(\mathrm{R})}, \theta_{k, n}\right)=\min _{1 \leq k \leq K} f_{k}\left(q, \widetilde{p}_{k, n}^{(\mathrm{S})}, \widetilde{p}_{k, n}^{(\mathrm{R})}, \theta_{k, n}\right), \\
f^{(2)}(q)=\max _{\left(\widetilde{\mathbf{P}}^{(\mathrm{S})}, \widetilde{\mathbf{P}}^{(\mathrm{R})}, \Theta\right) \in \mathcal{A}} f^{(1)}\left(q, \widetilde{p}_{k, n}^{(\mathrm{S})}, \widetilde{p}_{k, n}^{(\mathrm{R})}, \theta_{k, n}\right) .
\end{array}
$$

Lemma 1. For finite $q^{*}$ values, i.e., $q^{*}<\infty$, we have (a) $q^{*} \geq q$ if and only if $f^{(2)}(q) \geq 0$,

(b) $q^{*} \leq q$ if and only if $f^{(2)}(q) \leq 0$,

(c) $q^{*}=q$ if and only if $f^{(2)}(q)=0$.

Proof: see Appendix C.

\section{B. Dual min - max Problem}

The generalized dual min - max problem $\mathfrak{D}$ corresponding to the primal max - min problem $\mathfrak{P}$ is written as

$$
\begin{aligned}
& \mathfrak{D}: \min _{1 \leq k \leq K} \max _{\widetilde{\mathbf{P}}(\mathrm{S})}, \widetilde{\mathbf{P}}^{(\mathrm{R})}, \Theta \frac{\widetilde{r}_{k}\left(\widetilde{p}_{k, n}^{(\mathrm{S})}, \widetilde{p}_{k, n}^{(\mathrm{R})}, \theta_{k, n}\right)}{\widetilde{p}_{k}\left(\widetilde{p}_{k, n}^{(\mathrm{S})}, \theta_{k, n}\right)} \\
& \text { s.t. } \widetilde{\mathrm{C} 1}, \widetilde{\mathrm{C}} 2, \widetilde{\mathrm{C}} 3, \widetilde{\mathrm{C}} 4, \widetilde{\mathrm{C}} 5, \widetilde{\mathrm{C}} 6 .
\end{aligned}
$$

\footnotetext{
${ }^{3}$ The value of $q$ can be updated using a primal Dinkelbach algorithm similar to the dual Dinkelbach algorithm presented in Section IV.
}

Generally speaking, $q^{*} \leq \pi^{*}$ [29], where $\pi^{*}=$ $\min _{1 \leq k \leq K} \max _{\widetilde{\mathbf{P}}^{(\mathrm{S})}, \widetilde{\mathbf{P}}^{(\mathrm{R})}, \Theta} \frac{\widetilde{r}_{k}\left(\widetilde{p}_{k, n}^{(\mathrm{S})}, \widetilde{p}_{k, n}^{(\overline{\mathrm{R}})}, \theta_{k, n}\right)}{\widetilde{p}_{k}\left(\widetilde{p}_{k, n}^{\mathrm{S})}, \theta_{k, n}\right)}$ is the optimal objective function value of the dual problem $\mathfrak{D}$. In other words, the solution of fractional dual min - max problem $\mathfrak{D}$ is an upper bound to the solution of the fractional primal $\max -\min$ problem $\mathfrak{P}$. In the following we find the sufficient conditions under which $\pi^{*}=q^{*}$, i.e., the solution of the dual problem $\mathfrak{D}$ is the same as the solution to the primal problem $\mathfrak{P}$.

Definition 1. The function $g: \mathbb{R}^{m} \times \mathbb{R}^{\ell} \rightarrow[-\infty, \infty]$ is called a concavelconvex bifunction on the convex set $\mathcal{X} \times \mathcal{Y}$ with $\mathcal{X} \subseteq$ $\mathbb{R}^{m}$ and $\mathcal{Y} \subseteq \mathbb{R}^{\ell}$ if for every $y \in \mathcal{Y}$ the function $x \rightarrow g(x, y)$ is concave on $\mathcal{X}$ and for every $x \in \mathcal{X}$ the function $y \rightarrow g(x, y)$ is convex on $\mathcal{Y}$. Moreover, a function $g: \mathbb{R}^{m} \times \mathbb{R}^{\ell} \rightarrow[-\infty, \infty]$ is called a convex/concave bifunction on $\mathcal{X} \times \mathcal{Y}$ if $-g$ is a concave/convex bifunction on the same set. A function $g$ : $\mathbb{R}^{m} \times \mathbb{R}^{\ell} \rightarrow[-\infty, \infty]$ is called an affine/affine bifunction if it is both a concave/convex and a convex/concave bifunction [30].

Under Definition 1, the following Theorem shows that the duality gap is zero between the primal $\max -\min$ problem $\mathfrak{P}$ and the dual min - max problem $\mathfrak{D}$.

Theorem 1. $\pi^{*}=q^{*}$ if the nonempty set $\mathcal{B} \subseteq \mathbb{R}^{m}$ given by $\{1, \ldots, K\}, K<\infty$, is a compact convex set, the nonempty set $\mathcal{A} \subseteq \mathbb{R}^{\ell}$ defined by the constraints $\widetilde{\mathrm{C} 1}, \widetilde{\mathrm{C} 2}, \widetilde{\mathrm{C}} 3, \widetilde{\mathrm{C}} 4, \widetilde{\mathrm{C}} 5$, and $\widetilde{\mathrm{C} 6}$ is a closed convex set, and there exists open convex set $\mathcal{A}_{1} \times \mathcal{B}_{1}$ containing $\mathcal{A} \times \mathcal{B}$ such that $\widetilde{p}_{k}\left(\widetilde{p}_{k, n}^{(\mathrm{S})}, \theta_{k, n}\right)$ is a positive finitevalued convex/concave bifunction and $\widetilde{r}_{k}\left(\widetilde{p}_{k, n}^{(\mathrm{S})}, \widetilde{p}_{k, n}^{(\mathrm{R})}, \theta_{k, n}\right)$ is a positive finite-valued concave/convex bifunction on $\mathcal{A}_{1} \times \mathcal{B}_{1}$. If $\widetilde{p}_{k}\left(\widetilde{p}_{k, n}^{(\mathrm{S})}, \theta_{k, n}\right)$ is a positive affine/affine bifunction, then $\widetilde{r}_{k}\left(\widetilde{p}_{k, n}^{(\mathrm{S})}, \widetilde{p}_{k, n}^{(\mathrm{R})}, \theta_{k, n}\right)$ is a finite-valued concave/convex bifunction.

Proof: see Appendix D.

\section{Dual Problem Solution \& Proposed Schemes}

In this section, we analyze and solve the dual min - max optimization problem using concepts from fractional programming and dual decompositions. We propose a modified Dinkelbach algorithm, named dual Dinkelbach, to achieve the optimal solution of the dual problem $\mathfrak{D}$ with a polynomial time complexity. We further exploit the inherent structure of the optimal solution and propose a low complexity suboptimal heuristic to balance between the complexity and the achieved performance.

\section{A. Solution of the Dual min - max Problem}

1) Fractional programming: The dual min-max fractional problem $\mathfrak{D}$ is transformed to a parametric form using concepts of fractional programming, where dual decomposition techniques can be used to solve the parametric dual problem. In particular, one can show that [27], [31]

$$
\begin{gathered}
\pi^{*}=\min _{1 \leq k \leq K} \max _{\widetilde{\mathbf{P}}^{(\mathrm{S})}, \widetilde{\mathbf{P}}^{(\mathrm{R})}, \Theta} \frac{\widetilde{r}_{k}\left(\widetilde{p}_{k, n}^{(\mathrm{S})}, \widetilde{p}_{k, n}^{(\mathrm{R})}, \theta_{k, n}\right)}{\widetilde{p}_{k}\left(\widetilde{p}_{k, n}^{(\mathrm{S})}, \theta_{k, n}\right)}, \\
\text { s.t. } \widetilde{\mathrm{C} 1}, \widetilde{\mathrm{C}} 2, \widetilde{\mathrm{C}} 3, \widetilde{\mathrm{C}} 4, \widetilde{\mathrm{C}} 5, \widetilde{\mathrm{C}} 6 .
\end{gathered}
$$




$$
\begin{aligned}
& \min _{\lambda^{(\mathrm{R})} \geq 0, \boldsymbol{\lambda}^{(\mathrm{S})} \geq 0, \boldsymbol{\lambda}^{(\widetilde{r})} \geq 0} \max _{\widetilde{\mathbf{P}}^{(\mathrm{S})}, \widetilde{\mathbf{P}}^{(\mathrm{R})}, \boldsymbol{\Theta}} L\left(\lambda^{(\mathrm{R})}, \boldsymbol{\lambda}_{k}^{(\mathrm{S})}, \boldsymbol{\lambda}_{k}^{(\widetilde{r})}, \widetilde{p}_{k, n}^{(\mathrm{S})}, \widetilde{p}_{k, n}^{(\mathrm{R})}, \theta_{k, n}\right), \\
& L\left(\lambda^{(\mathrm{R})}, \boldsymbol{\lambda}_{k}^{(\mathrm{S})}, \boldsymbol{\lambda}_{k}^{(\widetilde{r})}, \widetilde{p}_{k, n}^{(\mathrm{S})}, \widetilde{p}_{k, n}^{(\mathrm{R})}, \theta_{k, n}\right)= B \sum_{n=1}^{N} \theta_{k, n} \log _{2}\left(1+\frac{1}{\Gamma \theta_{k, n}}\left(\widetilde{p}_{k, n}^{(\mathrm{S})} \gamma_{k, n}^{(\mathrm{SD})}+\frac{\widetilde{p}_{k, n}^{(\mathrm{S})} \gamma_{k, n}^{(\mathrm{SR})} \widetilde{p}_{k, n}^{(\mathrm{R})} \gamma_{n}^{(\mathrm{RD})}}{\left.\left.\widetilde{p}_{k, n}^{(\mathrm{S})} \gamma_{k, n}^{(\mathrm{SR})}+\widetilde{p}_{k, n}^{(\mathrm{R})} \gamma_{n}^{(\mathrm{RD})}\right)\right)}\right.\right. \\
&-\pi\left(p_{k, \mathrm{c}}^{(\mathrm{S})}+\frac{1}{\xi_{k}^{(\mathrm{S})}} \sum_{n=1}^{N} \widetilde{p}_{k, n}^{(\mathrm{S})}\right)+\lambda^{(\mathrm{R})}\left(p_{\max }^{(\mathrm{R})}-\sum_{k=1}^{N} \sum_{n=1}^{N} \widetilde{p}_{k, n}^{(\mathrm{R})}\right) \\
&+\sum_{k=1}^{K} \lambda_{k}^{(\mathrm{S})}\left(P_{k, \max }^{(\mathrm{S})}-\sum_{n=1}^{N} \widetilde{p}_{k, n}^{(\mathrm{S})}\right)+\sum_{n=1}^{N} \lambda_{k}^{(\widetilde{r})}\left(\widetilde{r}_{k}\left(\widetilde{p}_{k, n}^{(\mathrm{S})}, \widetilde{p}_{k, n}^{(\mathrm{R})}, \theta_{k, n}\right)-r_{k, \max }\right),
\end{aligned}
$$

$$
\begin{aligned}
& \text { if and only if } \\
& \begin{array}{r}
\min _{1 \leq k \leq K} \max _{\widetilde{\mathbf{P}}^{(\mathrm{S})}, \widetilde{\mathbf{P}}^{(\mathrm{R})}, \Theta} \widetilde{r}_{k}\left(\widetilde{p}_{k, n}^{(\mathrm{S})}, \widetilde{p}_{k, n}^{(\mathrm{R})}, \theta_{k, n}\right)-\pi^{*} \widetilde{p}_{k}^{(\mathrm{S})}\left(\widetilde{p}_{k, n}^{(\mathrm{S})}, \theta_{k, n}\right) \\
=\widetilde{r}_{k^{*}}\left(\widetilde{p}_{k, n}^{*,(\mathrm{~S})}, \widetilde{p}_{k, n}^{*,(\mathrm{R})}, \theta_{k, n}^{*}\right)-\pi^{*} \widetilde{p}_{k^{*}}^{(\mathrm{S})}\left(\widetilde{p}_{k, n}^{*,(\mathrm{~S})}, \theta_{k, n}^{*}\right)=0 \\
\quad \text { s.t. } \widetilde{\mathrm{C} 1}, \widetilde{\mathrm{C} 2}, \widetilde{\mathrm{C}} 3, \widetilde{\mathrm{C}} 4, \widetilde{\mathrm{C} 5}, \widetilde{\mathrm{C}} 6,
\end{array}
\end{aligned}
$$

where $\left(\widetilde{p}_{k, n}^{*,(\mathrm{~S})}, \widetilde{p}_{k, n}^{*,(\mathrm{R})}, \theta_{k, n}^{*}\right)$ reflects the optimal auxiliary MS $k$ power, auxiliary RS power, and subcarrier assignment variables and $\widetilde{r}_{k *}$ and $\widetilde{p}_{k *}^{(\mathrm{S})}$ denote the MS transmission rate and transmit power, respectively, at the optimal value of $k$, i.e., $k^{*}$. In other words, there exists a parametric non-fractional optimization problem that is equivalent to the fractional optimization problem $\mathfrak{D}$ at $\pi^{*}$ and $\left(\widetilde{p}_{k, n}^{*,(\mathrm{~S})}, \widetilde{p}_{k, n}^{*,(\mathrm{R})}, \theta_{k, n}^{*}\right)$ and $k^{*}$. The proof is similar to the one given in [31] and it will not be provided due to space limitations. We propose a modified Dinkelbach algorithm, named dual Dinkelbach, to update $\pi$ and reach the optimal solution in Section IV-B.

As shown in Appendix A, $\widetilde{r}_{k}\left(\widetilde{p}_{k, n}^{(\mathrm{S})}, \widetilde{p}_{k, n}^{(\mathrm{R})}, \theta_{k, n}\right)$ and $\widetilde{p}_{k}^{(\mathrm{S})}\left(\widetilde{p}_{k, n}^{(\mathrm{S})}, \theta_{k, n}\right)$ are concave and affine, respectively, with respect to the auxiliary MSs power, auxiliary RS powers, and relaxed subcarrier assignment and is affine. Thus, the inner optimization problem in (22) at certain value of $\pi$ is concave and can be solved by convex optimization techniques. Assuming the existence of an interior point, the duality gap between the inner optimization problem in (22) and its dual is zero and strong duality holds [28]. That said, the dual of the problem in (22) is expressed as in (23) on the top of this page, where $L$ is the partial Lagrangian given as in (24) on the top of this page, and $\lambda^{(\mathrm{R})}, \lambda^{(\mathrm{S})}=\left[\lambda_{1}^{(\mathrm{S})}, \ldots, \lambda_{K}^{(\mathrm{S})}\right]^{\mathrm{T}}$, and $\boldsymbol{\lambda}^{(\widetilde{r})}=\left[\lambda_{1}^{(\widetilde{r})}, \ldots, \lambda_{K}^{(\widetilde{r})}\right]^{\mathrm{T}}$ are the non-negative Lagrangian multipliers associated with the constraints $\widetilde{\mathrm{C}} 1, \widetilde{\mathrm{C}} 2$, and $\widetilde{\mathrm{C}} 3$, respectively. Other constraints not considered in the partial Lagrangian function will be satisfied later.

At a certain value of $\pi$, (23) can be solved by dual decomposition via solving $K N$ subproblems to obtain the auxiliary MS and RS power allocations and subcarrier assignment from (24), and then by solving a master problem to update the Lagrange multipliers $\lambda^{(\mathrm{R})}, \boldsymbol{\lambda}^{(\mathrm{S})}, \boldsymbol{\lambda}^{(\widetilde{r})}$. The process continues until convergence or satisfying the constraints. The details of the dual decomposition process is outlined as follows.
2) Solving the $K N$ subproblems: At each iteration $t$ of the dual decomposition method and for a given initial values of the Lagrange multipliers $\lambda^{(\mathrm{R})}, \boldsymbol{\lambda}^{(\mathrm{S})}, \boldsymbol{\lambda}^{(\widetilde{r})}$, one can get the MSs and RS power and subcarrier allocations by solving the maximization part of the dual problem in (23). The first order optimality conditions, i.e., Karush-Kuhn-Tucker (KKT) are necessary and sufficient for optimality as the optimization problem is concave [28]. This is achieved by setting the gradient equal to zero at the optimal points as follows

$$
\begin{aligned}
& \frac{\partial L\left(\lambda^{(\mathrm{R})}, \boldsymbol{\lambda}_{k}^{(\mathrm{S})}, \boldsymbol{\lambda}_{k}^{(\widetilde{r})}, \tilde{p}_{k, n}^{(\mathrm{S})}, \widetilde{p}_{k, n}^{(\mathrm{R})}, \theta_{k, n}\right)}{\partial \widetilde{p}_{k, n}^{(\mathrm{S})}}=\left.0\right|_{\widetilde{p}_{k, n}^{(\mathrm{S})}=\widetilde{p}_{k, n}^{*,(\mathrm{~S})}} \\
& \frac{\partial L\left(\lambda^{(\mathrm{R})}, \boldsymbol{\lambda}_{k}^{(\mathrm{S})}, \boldsymbol{\lambda}_{k}^{(\widetilde{r})}, \widetilde{p}_{k, n}^{(\mathrm{S})}, \widetilde{p}_{k, n}^{(\mathrm{R})}, \theta_{k, n}\right)}{\partial \widetilde{p}_{k, n}^{(\mathrm{R})}}=\left.0\right|_{\widetilde{p}_{k, n}^{(\mathrm{R})}=\widetilde{p}_{k, n}^{*,(\mathrm{R})}}
\end{aligned}
$$

The optimal powers at the MS $k$ and the RS is given by

$p_{k, n}^{*,(\mathrm{~S})}=\left[\frac{B\left(1+\frac{\lambda_{k}^{(\widetilde{r})}}{2}\right)}{\Gamma \lambda^{(\mathrm{R})} \ln (2)} \frac{\gamma_{k, n}^{2,(\mathrm{SR})} \gamma_{n}^{(\mathrm{RD})}}{\left(\gamma_{k, n}^{(\mathrm{SR})}+\frac{\gamma_{n}^{(\mathrm{RD})}}{\Psi_{k, n}}\right)^{2}} \frac{1}{\alpha_{k, n}}-\frac{1}{\alpha_{k, n}}\right]^{+}$

$p_{k, n}^{*,(\mathrm{R})}=\frac{1}{\Psi_{k, n}} p_{k, n}^{*,(\mathrm{~S})}$,

$$
\alpha_{k, n}=\frac{1}{\Gamma}\left(\gamma_{k, n}^{(\mathrm{SD})}+\frac{\gamma_{k, n}^{(\mathrm{SR})} \gamma_{n}^{(\mathrm{RD})}}{\Psi_{k, n} \gamma_{k, n}^{(\mathrm{SR})}+\gamma_{n}^{(\mathrm{RD})}}\right),
$$

where $\Psi_{k, n}$ is defined as in (30) on the top of the next page. The details of the derivation of (27) and (28) are given in Appendix B. As can be seen, the optimal MS and RS powers, $p_{k, n}^{*,(\mathrm{~S})}$ and $p_{k, n}^{*,(\mathrm{R})}$, can be considered as waterfilling solutions with multiple waterlevels where $\alpha_{k, n}$ represents the effective channel gain of MS $k$ on subcarrier $n$. It is worthy to mention that non-negative power constraints in $\widetilde{\mathrm{C}} 4$ are taken into account for the optimal MS $k$ and RS powers by introducing the notation $[x]^{+}=\max (0, x)$. Using the first order optimality conditions, the optimal subcarrier assignment is calculated from (31) on the top of this page, where $\widetilde{p}_{k, n}^{*,(\mathrm{~S})}$ and $\widetilde{p}_{k, n}^{*,(\mathrm{R})}$ are obtained from (27) and (28), respectively. Thus, (31) can be further simplified as 


$$
\begin{aligned}
& \Psi_{k, n}=\frac{1+\sqrt{1+\left(1+\frac{\gamma_{k, n}^{(\mathrm{SR})}}{\gamma_{k, n}^{(\mathrm{SD})}}\right)\left(\frac{1}{\lambda^{(\mathrm{R})}}\left(\frac{\pi}{\xi_{k}^{(\mathrm{S})}}+\lambda_{k}^{(\mathrm{S})}\right) \frac{\gamma_{n}^{(\mathrm{RD})}}{\gamma_{k, n}^{(\mathrm{SD})}}-1\right)}}{\frac{\gamma_{k, n}^{(\mathrm{SR})}}{\gamma_{n}^{(\mathrm{RD})}}\left(\frac{1}{\lambda^{(\mathrm{R})}}\left(\frac{\pi}{\xi_{k}^{(\mathrm{S})}}+\lambda_{k}^{(\mathrm{S})}\right) \frac{\gamma_{n}^{\mathrm{RD})}}{\gamma_{k, n}^{(\mathrm{SD})}}-1\right)}, \\
& \left.-\frac{1}{\ln (2)} \frac{\frac{1}{\Gamma \theta_{k, n}}\left(\widetilde{p}_{k, n}^{*,(\mathrm{~S})} \gamma_{k, n}^{(\mathrm{SD})}+\frac{\widetilde{p}_{k, n}^{*,(\mathrm{~S})} \gamma_{k, n}^{(\mathrm{SR})} \widetilde{p}_{k, n}^{*,(\mathrm{R})} \gamma_{n}^{(\mathrm{RD})}}{\widetilde{p}_{k, n}^{(\mathrm{S})} \gamma_{k, n}^{(\mathrm{SR})}+\widetilde{p}_{k, n}^{*,(\mathrm{R})} \gamma_{n}^{(\mathrm{RD})}}\right)}{\left(1+\frac{1}{\Gamma \theta_{k, n}}\left(\widetilde{p}_{k, n}^{*,(\mathrm{~S})} \gamma_{k, n}^{(\mathrm{SD})}+\frac{\widetilde{p}_{k, n}^{*,(\mathrm{~S})} \gamma_{k, n}^{(\mathrm{SR})} \widetilde{p}_{k, n}^{*,(\mathrm{R})} \gamma_{n}^{(\mathrm{RD})}}{\widetilde{p}_{k, n}^{*,(\mathrm{~S})} \gamma_{k, n}^{(\mathrm{SR})}+\widetilde{p}_{k, n}^{*,(\mathrm{R})} \gamma_{n}^{(\mathrm{RD})}}\right)\right)}\right], \\
& \frac{\partial L\left(\lambda^{(\mathrm{R})}, \boldsymbol{\lambda}_{k}^{(\mathrm{S})}, \boldsymbol{\lambda}_{k}^{(\widetilde{r})}, \widetilde{p}_{k, n}^{(\mathrm{S})}, \widetilde{p}_{k, n}^{(\mathrm{R})}, \theta_{k, n}\right)}{\partial \theta_{k, n}} \\
& =B\left(1+\frac{\lambda_{k}^{(\widetilde{r})}}{2}\right)\left(\log _{2}\left(\Upsilon_{k, n}\right)-\frac{1}{\ln (2)} \frac{\Upsilon_{k, n}-1}{\left(\Upsilon_{k, n}\right)}\right), \\
& =\rho_{k, n} \begin{cases}<0, & \text { if } \theta_{k, n}^{*}=0, \\
=0, & \text { if } \theta_{\mathrm{k}, \mathrm{n}}^{*} \in(0,1), \\
>0, & \text { if } \theta_{k, n}^{*}=1,\end{cases}
\end{aligned}
$$$$
\frac{\partial L\left(\lambda^{(\mathrm{R})}, \boldsymbol{\lambda}_{k}^{(\mathrm{S})}, \boldsymbol{\lambda}_{k}^{(\widetilde{r})}, \widetilde{p}_{k, n}^{(\mathrm{S})}, \widetilde{p}_{k, n}^{(\mathrm{R})}, \theta_{k, n}\right)}{\partial \theta_{k, n}}=B\left(1+\frac{\lambda_{k}^{(\widetilde{r})}}{2}\right)\left[\log _{2}\left(1+\frac{1}{\Gamma \theta_{k, n}}\left(\widetilde{p}_{k, n}^{*,(\mathrm{~S})} \gamma_{k, n}^{(\mathrm{SD})}+\frac{\widetilde{p}_{k, n}^{*,(\mathrm{~S})} \gamma_{k, n}^{(\mathrm{SR})} \widehat{p}_{k, n}^{*,(\mathrm{R})} \gamma_{n}^{(\mathrm{RD})}}{\widetilde{p}_{k, n}^{*,(\mathrm{~S})} \gamma_{k, n}^{(\mathrm{SR})}+\widetilde{p}_{k, n}^{*,(\mathrm{R})} \gamma_{n}^{(\mathrm{RD})}}\right)\right)\right.
$$

where $\Upsilon_{k, n}=\frac{B}{\Gamma \lambda^{(\mathrm{R})} \ln (2)}\left(1+\frac{\lambda_{k}^{(\widetilde{k})}}{2}\right) \gamma_{n}^{(\mathrm{RD})}\left(1+\frac{\gamma_{n}^{(\mathrm{RD})}}{\Psi_{k, n} \gamma_{k, n}^{(\mathrm{S})}}\right)$ Following [8], one can show that (32) comes from the fact that if the optimal value of $\theta_{k, n}$ occurs at the boundary of the feasible region, then $L\left(\lambda^{(\mathrm{R})}, \boldsymbol{\lambda}_{k}^{(\mathrm{S})}, \boldsymbol{\lambda}_{k}^{(\widetilde{r})}, \widetilde{p}_{k, n}^{(\mathrm{S})}, \widetilde{p}_{k, n}^{(\mathrm{R})}, \theta_{k, n}\right)$ must be decreasing with the values of $\theta_{k, n}$ that approach the interior of the feasible region. But since each subcarrier may only be used for transmission to a single MS, each subcarrier $n$ is allocated to the specific MS $k$ having the highest value of $\rho_{k, n}$ in (32) to achieve the highest increase in $L\left(\lambda^{(\mathrm{R})}, \boldsymbol{\lambda}_{k}^{(\mathrm{S})}, \boldsymbol{\lambda}_{k}^{(\widetilde{r})}, \widetilde{p}_{k, n}^{(\mathrm{S})}, \widetilde{p}_{k, n}^{\mathrm{R})}, \theta_{k, n}\right)$. That said, the optimal subcarrier assignment is written as

$$
\theta_{k, n}^{*}=\left\{\begin{array}{lc}
1, & \text { if } \rho_{k, n}=\max _{i} \rho_{i, n} \text { and } \rho_{k, n} \geq 0 \\
0, & \text { otherwise. }
\end{array}\right.
$$

As one can see, the constraints $\widetilde{\mathrm{C} 5}$ and $\widetilde{\mathrm{C} 6}$ that were not considered in the Lagrangian function in (24) are now satisfied.

3) Solving the master problem: Equations (27), (28), and (33) give a unique solution to the inner optimization problem in (23), hence, the dual problem in (23) is differentiable and the gradient method [24], [28] can be used to update the dual variables $\lambda^{(\mathrm{R})}, \boldsymbol{\lambda}^{(\mathrm{S})}=\left[\lambda_{1}^{(\mathrm{S})}, \ldots, \lambda_{K}^{(\mathrm{S})}\right]^{\mathrm{T}}$, and $\boldsymbol{\lambda}^{(\widetilde{r})}=$ $\left[\lambda_{1}^{(\widetilde{r})}, \ldots, \lambda_{K}^{(\widetilde{r})}\right]^{\mathrm{T}}$ as follows.

$$
\lambda^{(\mathrm{R})}(t+1)=\lambda^{(\mathrm{R})}(t)-\delta_{\lambda^{(\mathrm{R})}}(t)\left(p_{\max }^{(\mathrm{R})}-\sum_{k=1}^{K} \sum_{n=1}^{N} \widetilde{p}_{k, n}^{(\mathrm{R})}\right)
$$$$
\lambda_{k}^{(\mathrm{S})}(t+1)=\lambda_{k}^{(\mathrm{S})}(t)-\delta_{\lambda_{k}^{(\mathrm{S})}}(t)\left(p_{k, \max }^{(\mathrm{S})}-\sum_{n=1}^{N} \widetilde{p}_{k, n}^{(\mathrm{S})}\right),
$$

$$
\begin{aligned}
\lambda_{k}^{(\widetilde{r})}(t+1)= & \lambda_{k}^{(\widetilde{r})}(t)- \\
& \delta_{\lambda_{k}^{(\widetilde{r})}}(t)\left(\widetilde{r}_{k}\left(\widetilde{p}_{k, n}^{(\mathrm{S})}, \widetilde{p}_{k, n}^{(\mathrm{R})}, \theta_{k, n}\right)-r_{k, \max }^{(\mathrm{S})}\right),(36)
\end{aligned}
$$

where $\delta_{\lambda^{(\mathrm{R})}}, \delta_{\lambda_{k}^{(\mathrm{S})}}(t)$, and $\delta_{\lambda_{k}^{(\widetilde{r})}}(t)$ are sufficiently small step sizes associated ${ }^{k}$ with calculating the Lagrangian multipliers $\lambda^{(\mathrm{R})}, \lambda_{k}^{(\mathrm{S})}$, and $\lambda_{k}^{(\widetilde{r})}$, respectively, that are optimized to obtain fast convergence and $t$ is the iteration index.

\section{B. Proposed Schemes and Complexity Analysis}

The proposed modified Dinkelbach algorithm, i.e., dual Dinkelbach algorithm, is formally expressed as follows.

\section{Dual Dinkelbach Algorithm}

1: Start with an initial $\pi, \lambda^{(\mathrm{R})}, \lambda_{k}^{(\mathrm{S})}$, and $\lambda_{k}^{(\widetilde{r})}$.

2: Find optimal $p_{k, n}^{*,(\mathrm{~S})}, p_{k, n}^{*,(\mathrm{R})}, \theta_{k, n}^{*}$ according to (27), (28), and (33), respectively.

3: Update $\lambda^{(\mathrm{R})}, \lambda_{k}^{(\mathrm{S})}$, and $\lambda_{k}^{(\widetilde{r})}$ according to (34), (35), and (36), respectively.

4: Go to step 2 until constraints are satisfied or certain stopping criterion is satisfied

5: Calculate $\eta_{k}$ according to (8) and find worst $\eta_{k}$ as $\min _{1 \leq k \leq K} \eta_{k}$.

6: Update $\pi$ with $\min _{1 \leq k \leq K} \eta_{k}$.

7: Repeat steps $2-5$ until convergence or certain stopping criterion is satisfied

The complexity of the proposed algorithm is analyzed as follows. At each iteration of the Dinkelbach's method to update $\pi$ and at each iteration of the dual decomposition method to update the Lagrangian multipliers, the optimal source and relay powers and subcarrier assignment is found according to (27), (28), and (33), respectively. This is of computational complexity of $\mathcal{O}(K N)$. Given that the number of iterations to reach convergence for the dual decomposition and Dinkelback methods are $I_{\text {decomposition }}$ and $I_{\text {Dinkelback }}$, respectively, we can conclude that the computational complexity of the dual Dinkelbach algorithm is $\mathcal{O}\left(I_{\text {Dinkelback }} I_{\text {decomposition }} K N\right)$. Given the separate power and transmission rate constraints of each MSs in the uplink optimization problem, the complexity is dominated by the number of iterations to reach convergence of dual decomposition methods. 
In the following, we exploit the inherent structure of the obtained multiple levels waterfilling solution and propose a low complexity suboptimal heuristic in order to balance achieved performance with computational complexity. The idea of the heuristic is to allocate the MSs and RS powers suboptimally based on the effective channel gain $\alpha_{k, n}$ given in (29) while meeting the MSs and RS power constraints as follows.

$$
\begin{aligned}
p_{k, n}^{\mathrm{sub},(\mathrm{S})} & =\frac{\alpha_{k, n}}{\sum_{n=1}^{N} \alpha_{k, n}} p_{k, \text { max }}^{(\mathrm{S})}, \\
p_{k, n}^{\mathrm{sub},(\mathrm{R})} & =\frac{1}{\Psi_{k, n}} \frac{\alpha_{k, n}}{\sum_{n=1}^{N} \alpha_{k, n}} p_{k, \max }^{(\mathrm{R})} .
\end{aligned}
$$

In the low complexity suboptimal heuristic, subcarriers are allocated in a greedy manner to maximize the worst-EE, i.e., maximize the EE of the MS with the lowest EE value. More specifically, subcarrier $n^{\prime}$ is assigned to worst-EE MS $k^{\prime}$ if and only if its transmission rate constraint is not satisfied or if and only if its transmission rate constraint is satisfied and the EE of MS $k^{\prime}$ increases after using this subcarrier. Additionally, once subcarrier $n^{\prime}$ is allocated to MS $k^{\prime}$, then for all other MSs, i.e., $k \neq k^{\prime}$, the MSs and RS powers on subcarrier $n^{\prime}$ are set to zero and the power budget is redistributed on the remaining subcarriers. The process repeats until convergence or certain stopping criterion is met. The low complexity suboptimal heuristic is summarized as follows.

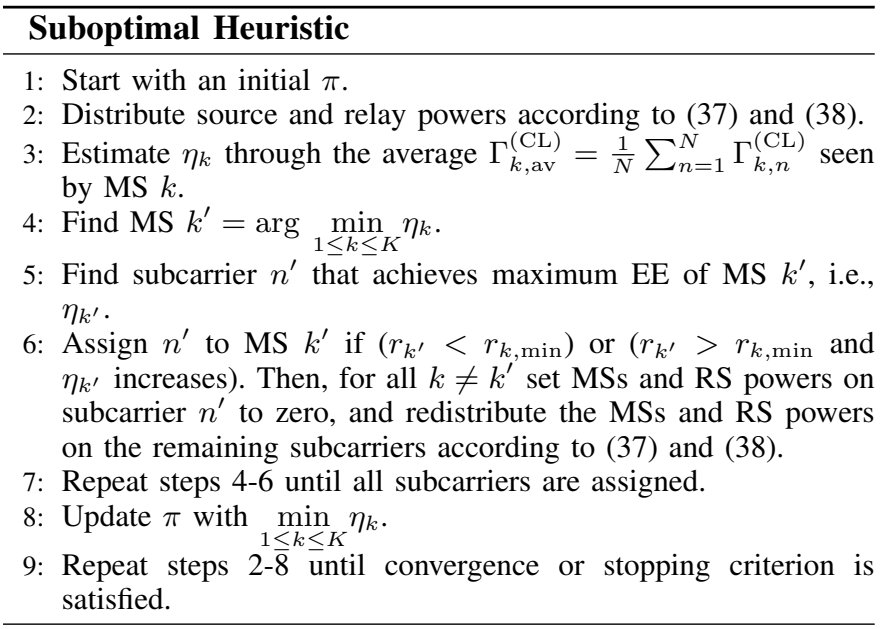

As can be seen, the complexity of finding the MSs and RS powers according to (37) and (38), respectively, is $\mathcal{O}(K N)$. The complexity of estimating $\eta_{k}$, finding MS $k^{\prime}$ with worst EE, and finding subcarrier $n^{\prime}$ that achieves maximum EE of MS $k^{\prime}$ are $\mathcal{O}(N), \mathcal{O}(K)$, and $\mathcal{O}(N)$ respectively. The complexity of Step 6 is $\mathcal{O}(K N)$. Since Steps 4-6 repeat at most $N$ times, then the complexity is $\mathcal{O}(N(N+K+N+K N))=$ $\mathcal{O}\left(K N^{2}\right)$. Hence, we can conclude that the complexity of the whole suboptimal heuristic is $\mathcal{O}\left(I_{\text {Dinkelback }} K N^{2}\right)$. As can be seen, the complexity of the suboptimal scheme is lower than its counterpart of the dual Dinkelbach algorithm given that $I_{\text {decomposition }}>>N$.

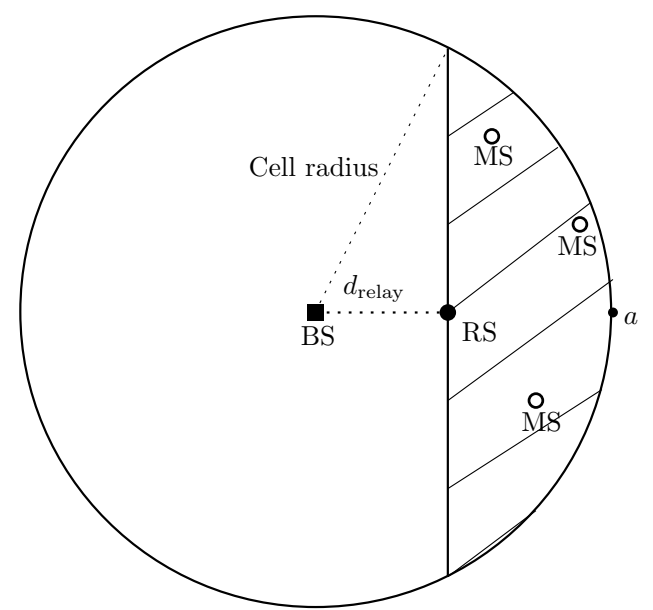

Fig. 2: Base station, relay, and mobile stations positioning in cellular network.

\section{Simulation Results}

\section{A. Simulation Setup}

In this section, in order to demonstrate the effectiveness of the subcarrier and power allocation schemes, we present simulation results. We consider the cellular network in Fig. 2, where $K \mathrm{MSs}$ are to communicate with a single BS through the assistance of a RS. The BS is assumed to be in the center of the cell and the normalized distance between the BS and the RS to the cell radius is denoted as $d_{\text {relay }}$. The RS position is assumed to change on the line between the BS and point $a$ at the edge of the cell, i.e., the value of $d_{\text {relay }} \in(0$, cell radius $)$. Further, MSs are distributed uniformly in the shaded area. As such to obtain channel gains on the links between MSs-RS and RS-BS better than the channel gains on the link between MSs-BS as in [32]. The channel coefficients are independent distributed circularly symmetric complex Gaussian random variables and distributed as $\mathcal{C N}\left(0, N_{o} B\right)$, where $N_{o}$ is the noise power spectral density, and the log-distance path loss is considered. Unless otherwise mentioned, the simulation parameters are summarized in Table I. To evaluate the performance, we define and use the following metrics:

- worst-EE to denote the average EE of the MS with the lowest EE value over the number of channel realizations.

- best-EE to denote the average EE of the MS with the highest EE value over the number of channel realizations.

- global-EE to denote the average EE of the whole network over the number of channel realizations.

- average transmission rate to denote the average transmission rate of the whole network over the number of channel realizations.

Additionally, we use the well-known Jain's fairness index [33] to measure how fairly the resources are allocated to different MSs in terms of EE. The EE Jain's fairness index is defined as [33]

$$
\text { EE fairness index }=\frac{\left(\sum_{k=1}^{K} \eta_{k}\right)^{2}}{K \sum_{k=1}^{K} \eta_{k}^{2}}
$$


TABLE I: Simulation PARAMETERS

\begin{tabular}{l|l}
\hline Subcarrier bandwidth $(B)$ & $15 \mathrm{kHz}$ \\
\hline Number of subcarriers $(N)$ & 128 \\
\hline Noise power spectral density $\left(N_{o}\right)$ & $-174 \mathrm{dBm} / \mathrm{Hz}$ \\
\hline Path loss exponent & 4 \\
\hline Number of MSs $(K)$ & 3 \\
\hline Normalized relay location to cell radius $\left(d_{\text {relay }}\right)$ & 0.4 \\
\hline Cell radius & $1 \mathrm{~km}$ \\
\hline SNR gap $(\Gamma)$ & $0 \mathrm{~dB}$ \\
\hline MSs power amplifier efficiencies $\left(\xi_{k}^{(\mathrm{S})}\right), k \in\{1, \ldots, K\}[18]$ & $38 \%$ \\
\hline Fixed power consumptions $\left(p_{k, c}\right), k \in\{1, \ldots, K\}[18]$ & $100 \mathrm{~mW}$ \\
\hline MSs maximum transmit power $\left(p_{k, \max }^{(\mathrm{S})}\right), k \in\{1, \ldots, K\}$ & $20 \mathrm{dBm}$ \\
\hline Relay maximum transmit power $\left(p_{\max }^{(\mathrm{R})}\right), k \in\{1, \ldots, K\}$ & $10 \mathrm{dBW}$ \\
\hline MSs minimum supported transmission rate $\left(r_{k, \min }^{(\mathrm{S})}\right), k \in\{1, \ldots, K\}$ & $10 \mathrm{kbps}$ \\
\hline Number of channel realizations & 1000 \\
\hline
\end{tabular}

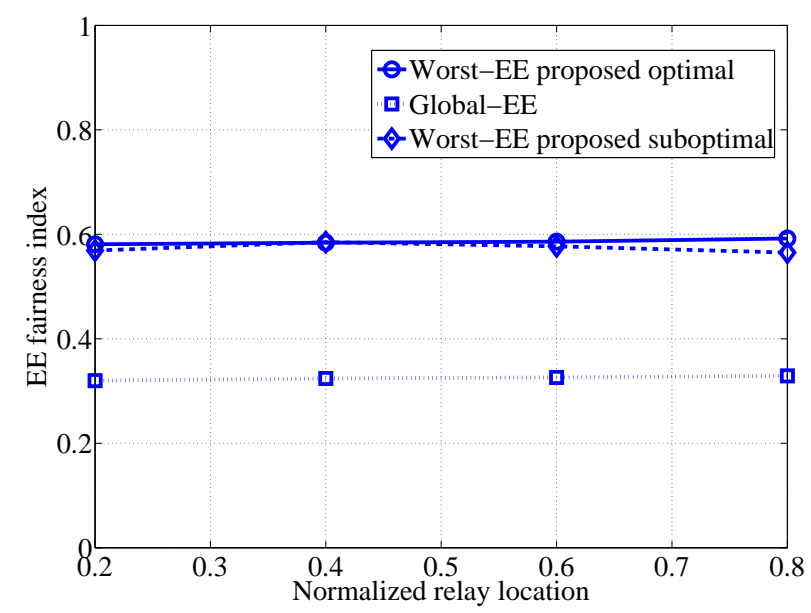

(a) EE fairness index.

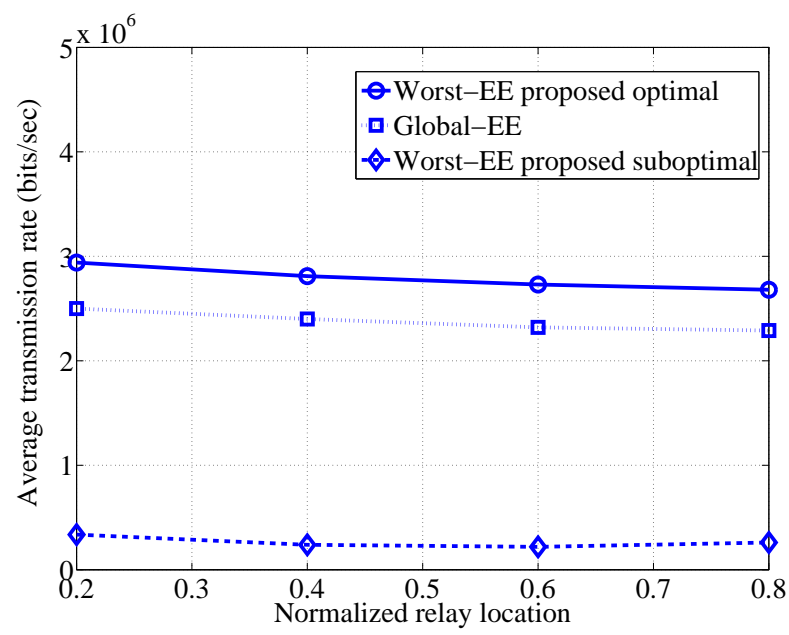

(b) Average transmission rate.

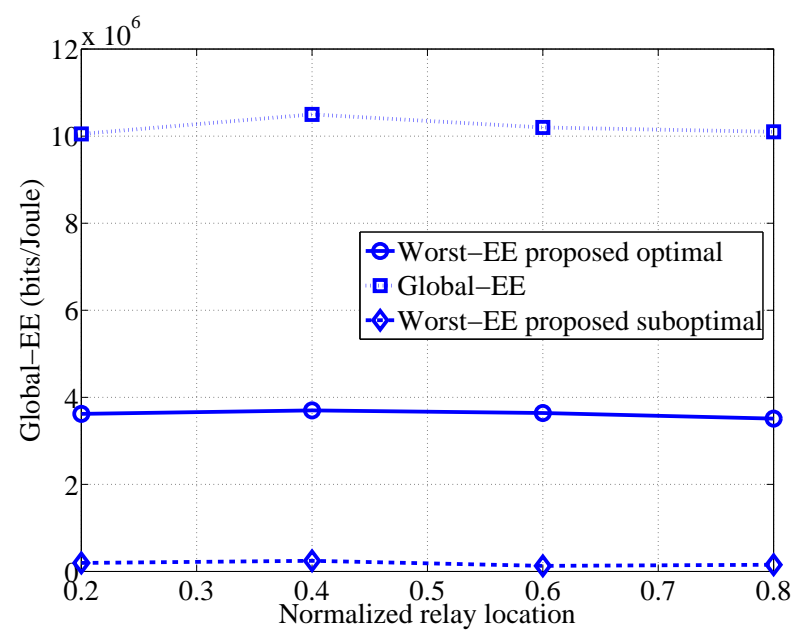

(c) Average EE.

Fig. 3: The effect of relay location on EE fairness index, average transmission rate, and global-EE.

with a value lies in the interval $\left[\frac{1}{K}, 1\right]$. In this interval, a value of $\frac{1}{K}$ corresponds to the least fair allocation between MSs in which only one MS receives non-zero benefits from the network. On the other hand, a value of 1 corresponds to the most fair allocation in which all MSs receive the same non-zero benefit from the network. To show the advantage and suitability of the worst-EE maximization for the uplink transmission scenario, we compare the performance of the 


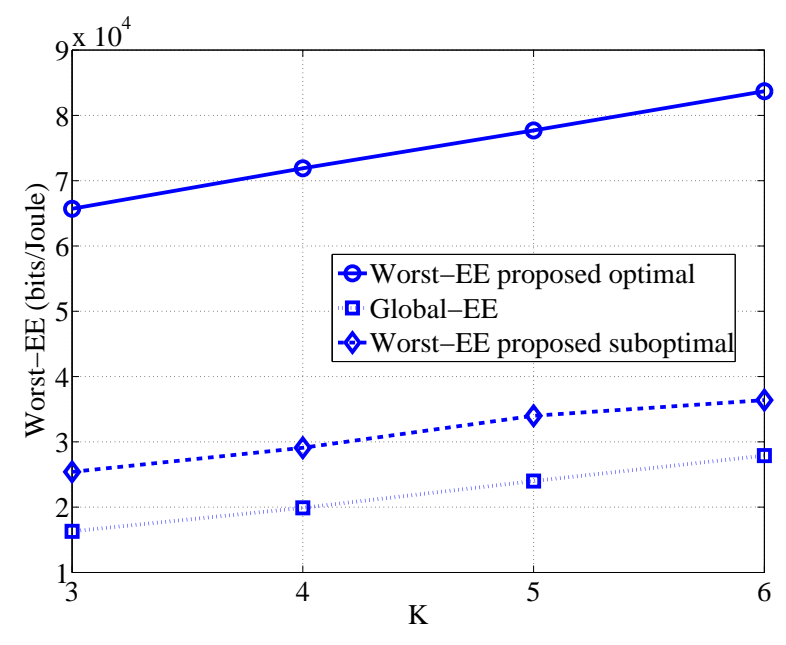

(a) Worst-EE.

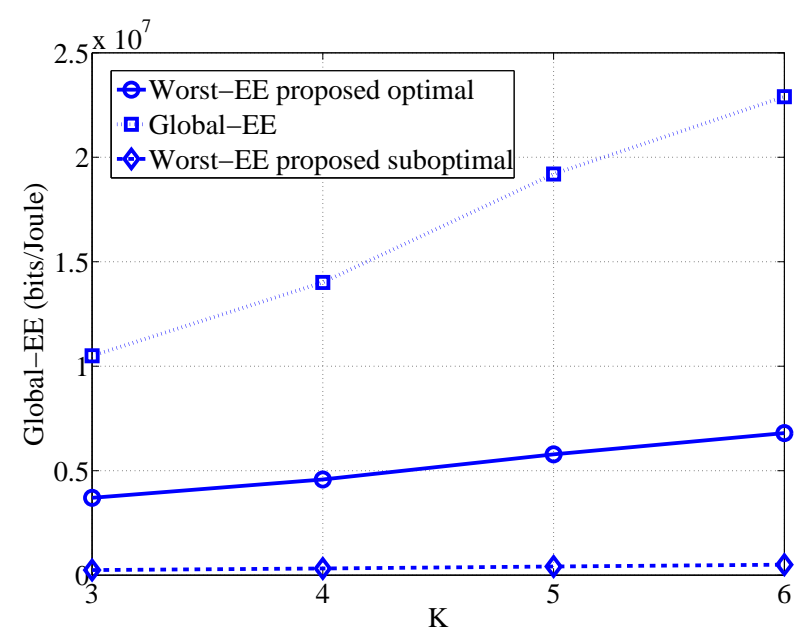

(c) Global-EE.

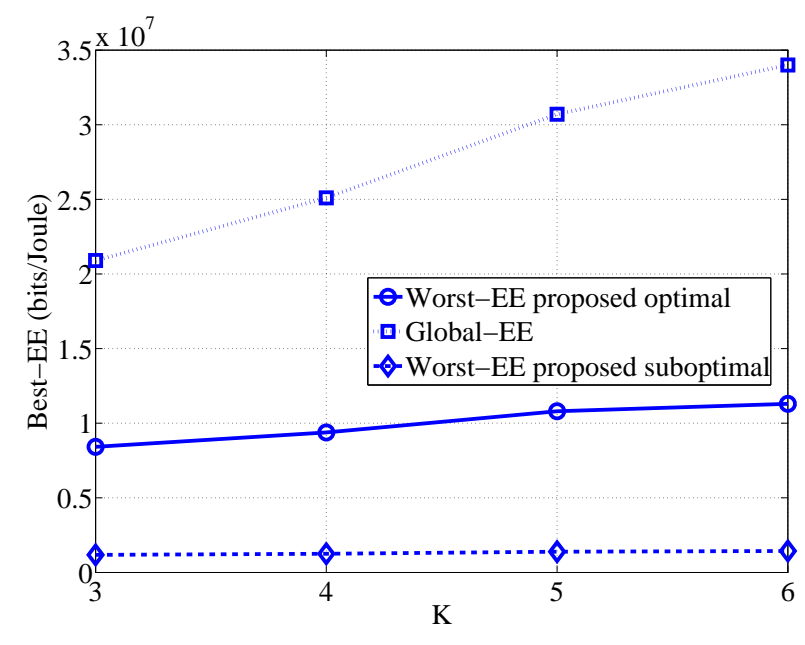

(b) Best-EE.

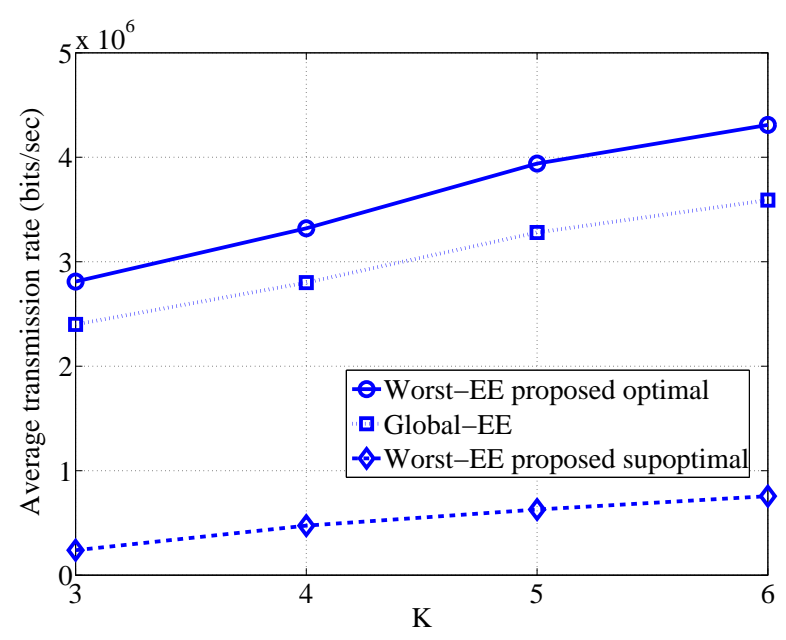

(d) Average transmission rate.

Fig. 4: The effect of number of MSs $K$ on the worst-EE, best-EE, global-EE, and average transmission rate.

proposed schemes to the global-EE maximization scheme [17] that is used for the downlink transmission, after matching the operating conditions, i.e., having separate MSs and RS power constraints.

\section{B. Effect of Relay Location}

Fig. 3 illustrates the effect of the relay location on the EE Jain's fairness index, average transmission rate, and globalEE. As can be seen, for the proposed schemes as well as the global-EE maximization scheme, the RS location within the cell slightly affects the system performance. One can see that the average transmission rate of the network slightly improves as the RS is placed close to the BS. This comes form the fact that the RS benefits from reduced path loss on the RS-BS link. However, placing the RS too close to the BS, results in higher transmit powers for the MSs and hence slightly reduces the global-EE. Interestingly, the EE Jain's fairness index is almost independent of the relative RS location which means that the resources allocated to different MSs and RS are relatively the same for different RS positions.
In Fig. 3a, both the proposed dual Dinkelbach algorithm and the the suboptimal heuristic improve the network fairness by almost $80 \%$ when compared to global-EE maximization scheme. This clearly reveals that the adopted worst-EE metric is suitable for the uplink transmission when compared to global-EE metric. Moreover, the proposed dual Dinkelbach algorithm improves the average network transmission rate when compared to global-EE optimal solution as can be seen in Fig. 3b. However, such improvements of the proposed dual Dinkelbach algorithm (in terms of fairness and average transmission rate) comes at the expense of a reduction of the global-EE of the whole network as seen in Fig. 3c. Fig. 3 reveals that the proposed suboptimal heuristic performs well to attain its target (i.e., fairness between MSs) with reduced computational complexity, however, this comes at the expense of a reduction in the global-EE and average rate of the 


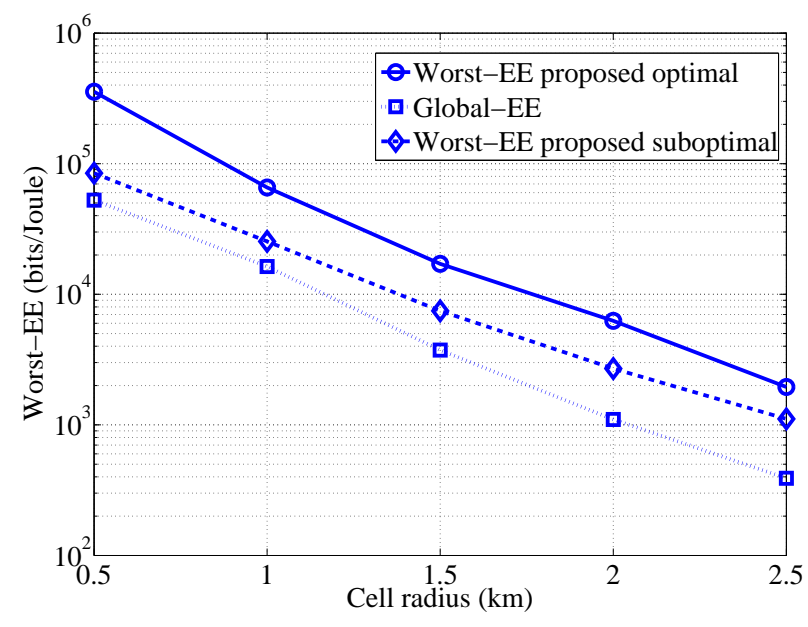

(a) Worst-EE.

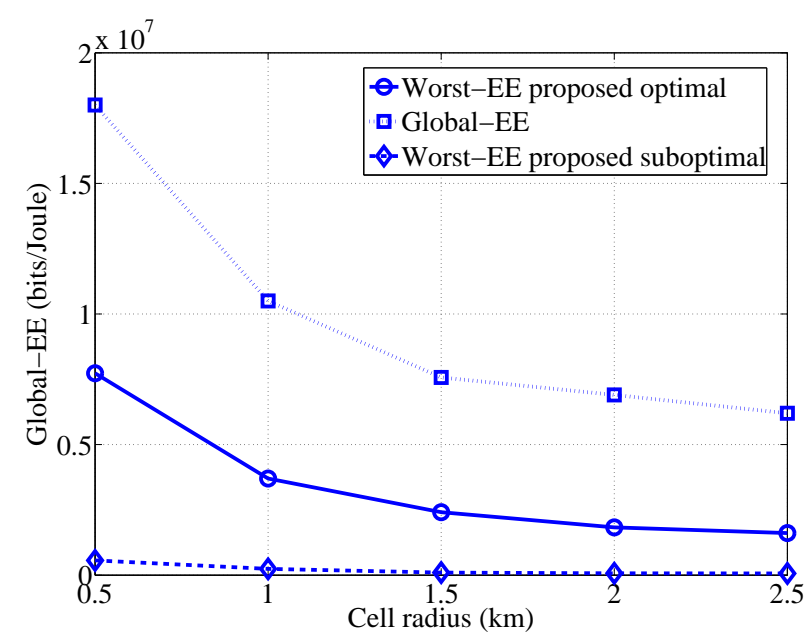

(c) Global-EE.

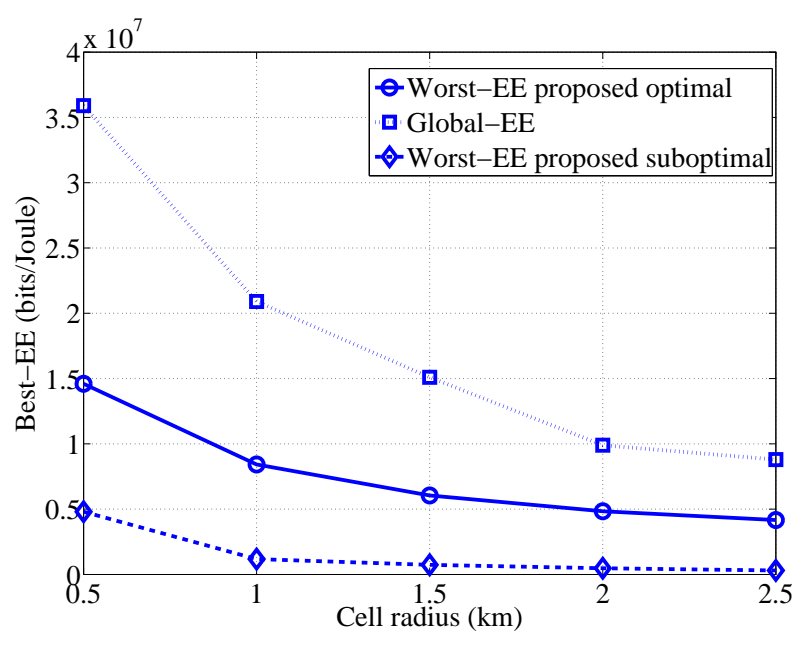

(b) Best-EE.

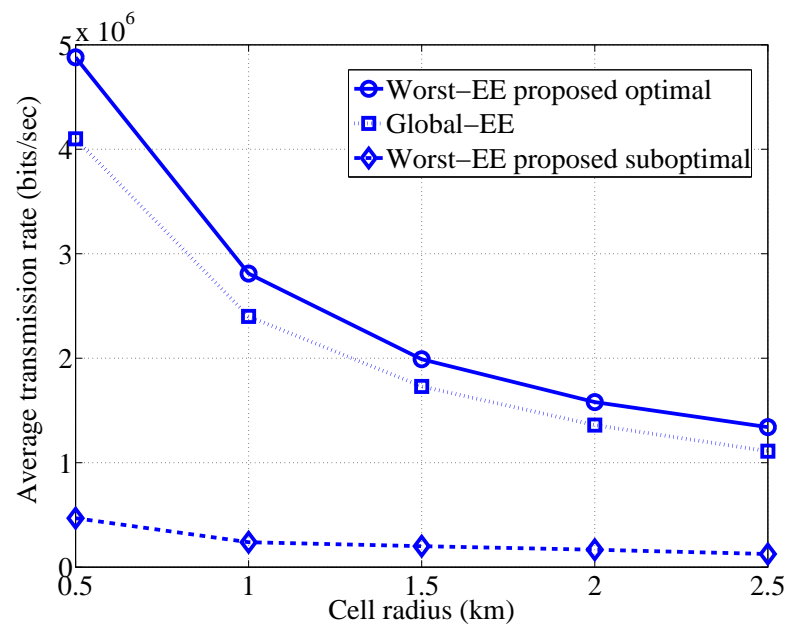

(d) Average transmission rate.

Fig. 5: The effect of cell radius on the worst-EE, best-EE, global-EE, and average transmission rate.

network ${ }^{4}$.

\section{Effect of Number of MSs K}

The EE Jain's fairness index reflects the individual EE of MSs. In the following, we show the worst-EE as another technique to quantify fairness. Additionally, global-EE and average transmission rate results are used to quantify the overall network performance, and best-EE results are presented for completeness of presentation. Fig. 4 presents the effect of the number of MSs $K$ on the worst-EE, best-EE, global-EE, and average transmission rate. As expected, as the number of MSs $K$ increases, the system performance (in terms of worst-EE, best-EE, global-EE, and average transmission rate) improves. This is due to the multi-user diversity, where subcarriers have better chance to be allocated to MSs that

\footnotetext{
${ }^{4}$ It is worthy to mention that other suboptimal schemes can be designed to improve the average transmission rate of the network, however, this comes at the expense of a deterioration of the worst-EE. Since the main target of the paper is to maximize worst-EE, and hence improve fairness, such suboptimal schemes are not discussed in the paper.
}

improve the performance. As can be seen in Fig. 4a, the proposed dual Dinkelbach algorithm significantly improves (almost 225\%) the worst-EE when compared to the reported works in the literature that maximize the global-EE, with the same computational complexity. Additionally, the sub-optimal heuristic improves the worst-EE by $42 \%$ when compared to optimal global-EE maximization, with reduced computational complexity. This clearly shows the effectiveness of the worstEE maximization for the uplink transmission in order to improve EE fairness between MSs. Additionally, the improvements of fairness between MSs, i.e., worst-EE, of the dualDinkelbach algorithm results in a corresponding increase in the average transmission rate of the network as shown in Fig. 4d. The improvements in worst-EE and average transmission rate come at the expense of a reduction in the global-EE and bestEE as can be seen in Figs. $4 c$ and $4 b$, respectively.

\section{Effect of Cell Radius}

In Fig. 5, the effect of the cell radius on worst-EE, best-EE, global-EE, and average transmission rate is plotted. As can 


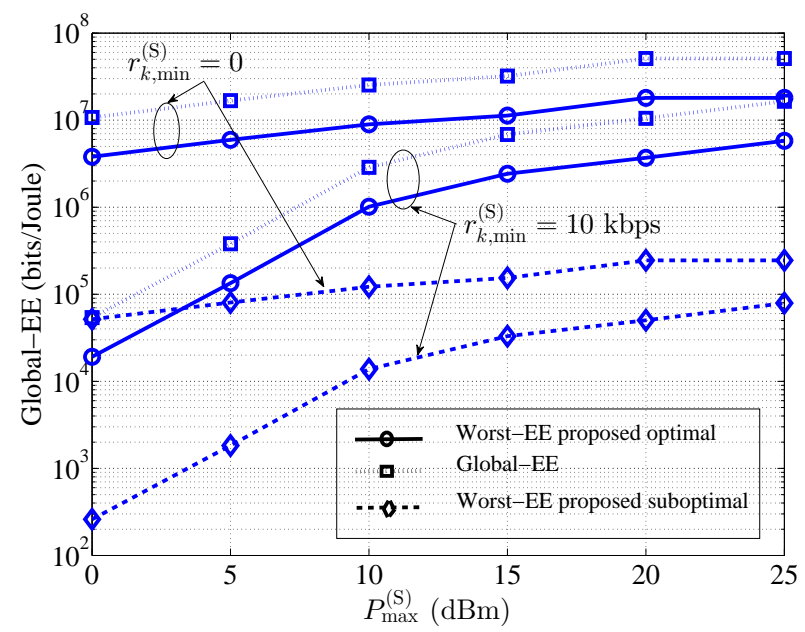

(a) Global-EE.

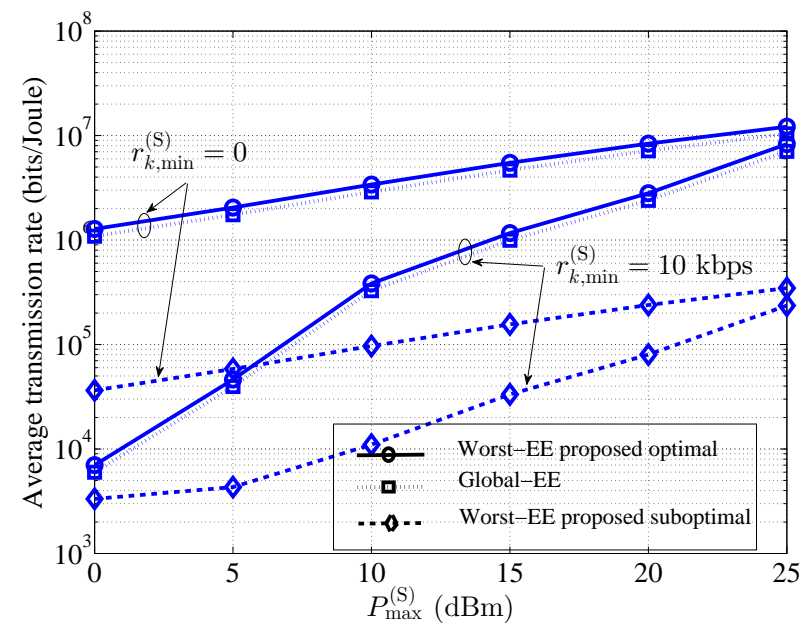

(b) Average transmission rate.

Fig. 6: The effect of MS maximum transmit power and minimum transmission rate constraints on the overall network performance (in terms of global-EE and average transmission rate).

be seen, as the cell radius increases the performance of the system deteriorates. This stems from the increased path loss between the communicating nodes, and hence, for the same MSs and RS power budgets the achieved transmission rate and EE per each MS are reduced. As expected, the proposed dual Dinkelbach and suboptimal schemes improve the worstEE by $303 \%$ and $61 \%$, respectively, compared to global-EE maximization (see Fig. 5a). Additionally, the improved fairness of the dual Dinkelbach's algorithm results in a better average transmission rate of the network, (see Fig. 5d). As expected, such improvements come at the expense of a reduction of the best-EE and global-EE of the network (see Figs. 5b and $5 c$, respectively). This emphasizes that worst-EE maximization introduced in this paper is more suitable of the uplink transmission when compared to the global-EE maximization that is traditionally used in the literature.

\section{E. Effect of MSs Transmit Power}

The effect of the MSs maximum transmit power on the global-EE and average transmission rate is depicted in Fig. 6 for the case of $r_{k, \min }^{(\mathrm{S})}=0$ and $10 \mathrm{kbps}$, respectively. As can be seen, increasing the MSs maximum transmit power increases both the average transmission rate and global-EE of the network. It is worthy noting that the global-EE saturates for higher values of MSs power budget. In other words, spending more power will only improve the transmission rate. At lower power budget, the minimum supported transmission rate is maintained at the expense of a reduction in the global-EE. As discussed earlier, the proposed dual Dinkelbach algorithm shows better average transmission rate when compared to global-EE maximization, while the global-EE maximization scheme achieve better EE for the whole network.

\section{F. Duality Gap Estimation}

In Fig. 7, we quantify the gap between the optimal solution of the mixed integer nonlinear fractional problem and the

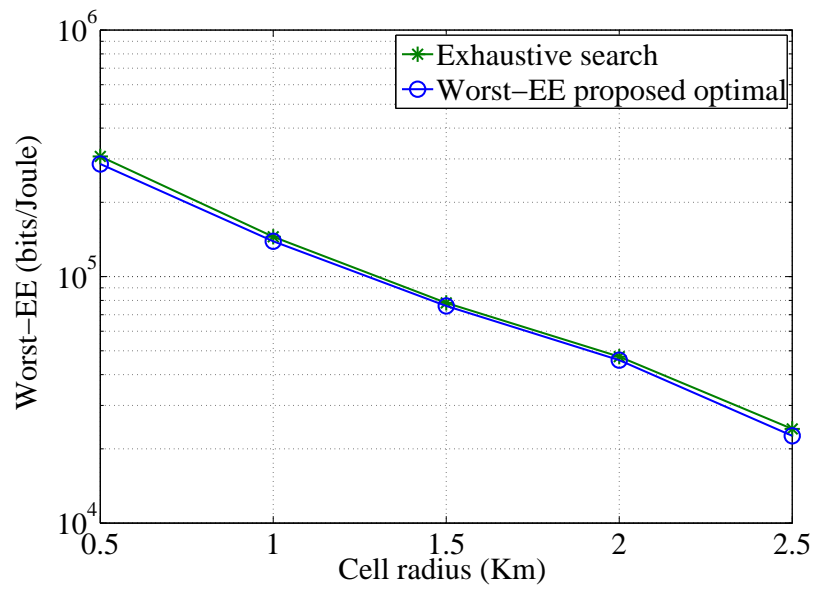

Fig. 7: Comparison of the exhaustive search and the proposed optimal algorithm in order to quantify the duality gap for $K=2$ and $N=4$.

corresponding relaxed problem for $K=2$ and $N=4$. Since the problem size is small, an exhaustive search algorithm is used to find the solution to the mixed integer nonlinear fractional problem. As can be seen from Fig. 7, the duality gap vanishes for as low as 4 subcarriers.

\section{CONCLUSION}

In this paper, we studied the problem of joint subcarrier and power allocation for the uplink scenario of $\mathrm{AF}$ cooperative OFDMA networks in order to maximize the worst-EE, i.e., to maximize the $\mathrm{EE}$ of the MS with the lowest $\mathrm{EE}$ value. The formulated primal max - min problem is computationally complex to solve even after relaxing the integer constraints on the subcarrier allocation. Therefore, we studied the structure of the primal problem and showed that the dual min - max problem attains the same optimal solution with polynomial time complexity. In order to further reduce the complexity, a suboptimal heuristic is proposed that exploits the structure 


$$
\begin{aligned}
& \frac{B\left(\frac{w_{k}}{\ln (2)}+\frac{\lambda_{k}^{(\widetilde{r})}}{2}\right)}{\frac{\pi}{\xi_{k}^{(\mathrm{S})}}+\lambda_{k}^{(\mathrm{S})}}\left(\gamma_{k, n}^{(\mathrm{SD})}\left(\widetilde{p}_{k, n}^{(\mathrm{S})} \gamma_{k, n}^{(\mathrm{SR})}+\widetilde{p}_{k, n}^{(\mathrm{R})} \gamma_{n}^{(\mathrm{RD})}\right)^{2}+\widetilde{p}_{k, n}^{2,(\mathrm{R})} \gamma_{k, n}^{2,(\mathrm{RD})} \gamma_{k, n}^{(\mathrm{SR})}\right) \\
& =\Gamma\left(1+\frac{1}{\Gamma \theta_{k, n}}\left(\widetilde{p}_{k, n}^{(\mathrm{S})} \gamma_{k, n}^{(\mathrm{SD})}+\frac{\widetilde{p}_{k, n}^{(\mathrm{S})} \gamma_{k, n}^{(\mathrm{SR})} \widetilde{p}_{k, n}^{(\mathrm{R})} \gamma_{n}^{(\mathrm{RD})}}{\widetilde{p}_{k, n}^{(\mathrm{S})} \gamma_{k, n}^{(\mathrm{SR})}+\widetilde{p}_{k, n}^{(\mathrm{R})} \gamma_{n}^{(\mathrm{RD})}}\right)\right)\left(\widetilde{p}_{k, n}^{(\mathrm{S})} \gamma_{k, n}^{(\mathrm{SR})}+\widetilde{p}_{k, n}^{(\mathrm{R})} \gamma_{n}^{(\mathrm{RD})}\right)^{2},
\end{aligned}
$$

$$
\begin{aligned}
& \frac{B\left(\frac{w_{k}}{\ln (2)}+\frac{\lambda_{k}^{(\widetilde{r})}}{2}\right)}{\lambda^{(\mathrm{R})}} \widetilde{p}_{k, n}^{2,(\mathrm{~S})} \gamma_{k, n}^{2,(\mathrm{SR})} \gamma_{n}^{(\mathrm{RD})} \\
& \quad=\Gamma\left(1+\frac{1}{\Gamma \theta_{k, n}}\left(\widetilde{p}_{k, n}^{(\mathrm{S})} \gamma_{k, n}^{(\mathrm{SD})}+\frac{\widetilde{p}_{k, n}^{(\mathrm{S})} \gamma_{k, n}^{(\mathrm{SR})} \widetilde{p}_{k, n}^{(\mathrm{R})} \gamma_{n}^{(\mathrm{RD})}}{\left.\left.\widetilde{p}_{k, n}^{(\mathrm{S})} \gamma_{k, n}^{(\mathrm{SR})}+\widetilde{p}_{k, n}^{(\mathrm{R})} \gamma_{n}^{(\mathrm{RD})}\right)\right)\left(\widetilde{p}_{k, n}^{(\mathrm{S})} \gamma_{k, n}^{(\mathrm{SR})}+\widetilde{p}_{k, n}^{(\mathrm{R})} \gamma_{n}^{(\mathrm{RD})}\right)^{2}}\right.\right.
\end{aligned}
$$

$$
\tilde{p}_{k, n}^{*,(\mathrm{~S})}=\tilde{p}_{k, n}^{*,(\mathrm{R})} \gamma_{n}^{(\mathrm{RD})} \frac{\sqrt{\gamma_{k, n}^{2,(\mathrm{SD})}-\left(\gamma_{k, n}^{(\mathrm{SD})}+\gamma_{k, n}^{(\mathrm{SR})}\right)\left(\gamma_{k, n}^{(\mathrm{SD})}-\frac{1}{\lambda_{k}^{(\mathrm{R})}}\left(\frac{\pi}{\xi_{k}^{(\mathrm{S})}}+\lambda_{k}^{(\mathrm{S})}\right) \gamma_{n}^{(\mathrm{RD})}\right)}-\gamma_{k, n}^{(\mathrm{SD})}}{\gamma_{k, n}^{(\mathrm{SR})}\left(\gamma_{k, n}^{(\mathrm{SD})}-\frac{1}{\lambda_{k}^{(\mathrm{R})}}\left(\frac{\pi}{\xi_{k}^{(\mathrm{S})}}+\lambda_{k}^{(\mathrm{S})}\right) \gamma_{n}^{(\mathrm{RD})}\right)},
$$

of the optimal solution. Numerical results confirmed that the worst-EE metric improves fairness between different MSs, and hence, it is suitable for uplink transmission scenarios compared to the global-EE that is traditionally used for EE maximization. More specifically, both the proposed schemes improve fairness between MSs by $80 \%$ when compared to global-EE maximization schemes. Additionally, the worst-EE is improved by up to $303 \%$ and $61 \%$ for the optimal and suboptimal schemes, respectively, when compared to globalEE maximization. Improvements in fairness, worst-EE, and average transmission rate come at a certain expense of the global-EE of the network.

\section{APPENDIX A}

Proof of The Quasi-Concavity of the ObJective Function of The PRimal max - min Problem $\mathfrak{P}$

One can show that the following function $f_{1}\left(\widetilde{p}_{k, n}^{(\mathrm{S})}, \widetilde{p}_{k, n}^{(\mathrm{R})}\right)=$ $\left(\widetilde{p}_{k, n}^{(\mathrm{S})} \gamma_{k, n}^{(\mathrm{SD})}+\frac{\widetilde{p}_{k, n}^{(\mathrm{S})} \gamma_{k, n}^{(\mathrm{SR})} \widetilde{p}_{k, n}^{(\mathrm{R})} \gamma_{n}^{(\mathrm{RD})}}{\widetilde{p}_{k, n}^{(\mathrm{S})} \gamma_{k, n}^{(\mathrm{SR})}+\widetilde{p}_{k, n}^{(\mathrm{R})} \gamma_{n}^{(\mathrm{RD})}}\right)$ is concave by showing that the Hessian is negative semi-definite and the eigenvalues are non-positive [28]. The Hessian of $f_{1}\left(\widetilde{p}_{k, n}^{(\mathrm{S})}, \widetilde{p}_{k, n}^{(\mathrm{R})}\right)$ can be found as

$$
\begin{aligned}
& \mathbf{H}\left(f_{1}\left(\widetilde{p}_{k, n}^{(\mathrm{S})}, \widetilde{p}_{k, n}^{(\mathrm{R})}\right)\right) \\
& =\frac{2 \gamma_{k, n}^{2,(\mathrm{SR})} \gamma_{k, n}^{2,(\mathrm{RD})}}{\left(\widetilde{p}_{k, n}^{(\mathrm{S})} \gamma_{k, n}^{(\mathrm{SR})}+\widetilde{p}_{k, n}^{(\mathrm{R})} \gamma_{n}^{(\mathrm{RD})}\right)^{3}}\left[\begin{array}{cc}
-\widetilde{p}_{k, n}^{2,(\mathrm{R})} & \widetilde{p}_{k, n}^{(\mathrm{S})} \widetilde{p}_{k, n}^{(\mathrm{R})} \\
\widetilde{p}_{k, n}^{(\mathrm{S})} \widetilde{p}_{k, n}^{(\mathrm{R})} & -\widetilde{p}_{k, n}^{2,(\mathrm{~S})}
\end{array}\right],
\end{aligned}
$$

and the eigenvalues can be calculated from $\operatorname{det}(\varpi \mathbf{I}-$ $\left.\mathbf{H}\left(f_{1}\left(\widetilde{p}_{k, n}^{(\mathrm{S})}, \widetilde{p}_{k, n}^{(\mathrm{R})}\right)\right)\right)=0$ as

$$
\begin{aligned}
& \varpi_{1}=0, \\
& \varpi_{2}=-\frac{2 \gamma_{k, n}^{2,(\mathrm{SR})} \gamma_{k, n}^{2,(\mathrm{RD})}\left(\widetilde{p}_{k, n}^{2,(\mathrm{R})}+\widetilde{p}_{k, n}^{2,(\mathrm{~S})}\right)}{\left(\widetilde{p}_{k, n}^{(\mathrm{S})} \gamma_{k, n}^{(\mathrm{SR})}+\widetilde{p}_{k, n}^{(\mathrm{R})} \gamma_{n}^{(\mathrm{RD})}\right)^{3}} .
\end{aligned}
$$

Clearly, the eigenvalues are non-positive, and hence, the Hessian matrix $\mathbf{H}\left(f_{1}\left(\widetilde{p}_{k, n}^{(\mathrm{S})}, \widetilde{p}_{k, n}^{(\mathrm{R})}\right)\right)$ is negative semi-definite and the function $f_{1}\left(\widetilde{p}_{k, n}^{(\mathrm{S})}, \widetilde{p}_{k, n}^{(\mathrm{R})}\right)$ is concave with respect to $\widetilde{p}_{k, n}^{(\mathrm{S})}$ and $\widetilde{p}_{k, n}^{(\mathrm{R})}$.

Since $f_{1}\left(\widetilde{p}_{k, n}^{(\mathrm{S})}, \widetilde{p}_{k, n}^{(\mathrm{R})}\right)$ is concave, hence, $1+f_{1}\left(\widetilde{p}_{k, n}^{(\mathrm{S})}, \widetilde{p}_{k, n}^{(\mathrm{R})}\right)$ is concave. Also, one can show that $f_{2}\left(\widetilde{p}_{k, n}^{(\mathrm{S})}, \widetilde{p}_{k, n}^{(\mathrm{R})}\right)=\log _{2}(1+$ $\left.f_{1}\left(\widetilde{p}_{k, n}^{(\mathrm{S})}, \widetilde{p}_{k, n}^{(\mathrm{R})}\right)\right)$ is concave using the composition property [28, pp. 84 , Eqn. (3.10)] as $f_{1}\left(\widetilde{p}_{k, n}^{(\mathrm{S})}, \widetilde{p}_{k, n}^{(\mathrm{R})}\right)$ is concave and $\log _{2}($. is concave and non-decreasing function. The perspective property of a function $f(x)$, which is defined as $g(x, t)=t f\left(\frac{x}{t}\right)$, maintains concavity [28]. That said, if $f_{2}\left(\widetilde{p}_{k, n}^{(\mathrm{S})}, \widetilde{p}_{k, n}^{(\mathrm{R})}\right)$ is concave, then $f_{3}\left(\widetilde{p}_{k, n}^{(\mathrm{S})}, \widetilde{p}_{k, n}^{(\mathrm{R})}, \theta_{k, n}\right)=\theta_{k, n} f_{2}\left(\frac{\widetilde{p}_{k, n}^{(\mathrm{S})}}{\theta_{k, n}}, \frac{\widetilde{p}_{k, n}^{(\mathrm{R})}}{\theta_{k, n}}\right)$ is also concave. Thus, $\widetilde{r}_{k}\left(\widetilde{p}_{k, n}^{(\mathrm{S})}, \widetilde{p}_{k, n}^{(\mathrm{R})}, \theta_{k, n}\right)$ is concave.

Additionally, $\widetilde{p}_{k}^{(\mathrm{S})}\left(\widetilde{p}_{k, n}^{(\mathrm{S})}, \theta_{k, n}\right)$ is affine and positive; hence, the objective function of the primal max $-\min$ problem $\mathfrak{P}$ is quasi-concave.

\section{APPENDIX B}

DERIVATION OF THE OPTIMAL POWER AND SUBCARRIER

\section{ALLOCATION}

From (25) and (26), one can respectively obtain (43) and (44) as in the top of this page. By dividing (43) and (44) one can relate $\widetilde{p}_{k, n}^{*,(\mathrm{~S})}$ and $\widetilde{p}_{k, n}^{*,(\mathrm{R})}$ as in (45) on the top of this page, then by substituting (45) into (44), one can obtain the optimal power of MS $k$ and RS at subcarrier $n$ as in (46) and (47) on the top of the next page. Accordingly, the final expressions for the optimal MS and relay powers are given as in (27) and (28), respectively. 


$$
\begin{aligned}
& p_{k, n}^{*,(\mathrm{~S})}=\left[\frac{\Gamma}{\gamma_{k, n}^{(\mathrm{SD})}+\frac{\gamma_{k, n}^{(\mathrm{SR})} \gamma_{n}^{(\mathrm{RD})}}{\left(\Psi_{k, n} \gamma_{k, n}^{(\mathrm{SR})}+\gamma_{n}^{(\mathrm{RD})}\right)}}\left(\frac{B\left(w_{k}+\frac{\lambda_{k}^{(\tilde{r})}}{2}\right)}{\Gamma \lambda^{(\mathrm{R})} \ln (2)} \frac{\gamma_{k, n}^{2,(\mathrm{SR})} \gamma_{n}^{(\mathrm{RD})}}{\left(\gamma_{k, n}^{(\mathrm{SR})}+\frac{\gamma_{n}^{(\mathrm{RD})}}{\Psi_{k, n}}\right)^{2}}-1\right)\right]^{+}, \\
& p_{k, n}^{*,(\mathrm{R})}=\left(\frac{1+\sqrt{1+\left(1+\frac{\gamma_{k, n}^{(\mathrm{SR})}}{\gamma_{k, n}^{(\mathrm{SD})}}\right)\left(\frac{1}{\lambda^{(\mathrm{R})}}\left(\frac{\pi}{\xi_{k}^{(\mathrm{S})}}+\lambda_{k}^{(\mathrm{S})}\right) \frac{\gamma_{n}^{(\mathrm{RD})}}{\gamma_{k, n}^{(\mathrm{SD})}}-1\right)}}{\frac{\gamma_{k, n}^{(\mathrm{SR})}}{\gamma_{n}^{(\mathrm{RD})}}\left(\frac{1}{\lambda^{(\mathrm{R})}}\left(\frac{\pi}{\xi_{k}^{(\mathrm{S})}}+\lambda_{k}^{(\mathrm{S})}\right) \frac{\gamma_{n}^{\mathrm{RD})}}{\gamma_{k, n}^{(\mathrm{SD})}}-1\right)}\right)^{-1} p_{k, n}^{*,(\mathrm{~S})}
\end{aligned}
$$

\section{APPENDIX C}

PROOF OF LEMMA 1

(a) - Since $q^{*}=\max _{\left(\widetilde{\mathbf{P}}^{(\mathrm{S})}, \widetilde{\mathbf{P}}^{(\mathrm{R})}, \boldsymbol{\Theta}\right) \in \mathcal{X}} q^{*}\left(\widetilde{p}_{k, n}^{(\mathrm{S})}, \widetilde{p}_{k, n}^{\mathrm{R})}, \theta_{k, n}\right)$, then there exists some feasible point

$\left(\widetilde{p}_{k, n}^{0,(\mathrm{~S})}, \widetilde{p}_{k, n}^{0,(\mathrm{R})}, \theta_{k, n}^{0}\right)$ such that

$$
q^{*}\left(\widetilde{p}_{k, n}^{0,(\mathrm{~S})}, \widetilde{p}_{k, n}^{0,(\mathrm{R})}, \theta_{k, n}^{0}\right)=\min _{1 \leq k \leq K} \frac{\widetilde{r}_{k}\left(\widetilde{p}_{k, n}^{0,(\mathrm{~S})}, \widetilde{p}_{k, n}^{0,(\mathrm{R})}, \theta_{k, n}^{0}\right)}{\widetilde{p}_{k}\left(\widetilde{p}_{k, n}^{0,(\mathrm{~S})}, \theta_{k, n}^{0}\right)} \geq q,
$$

which can be further expressed as

$$
\frac{\widetilde{r}_{k}\left(\widetilde{p}_{k, n}^{0,(\mathrm{~S})}, \widetilde{p}_{k, n}^{0,(\mathrm{R})}, \theta_{k, n}^{0}\right)}{\widetilde{p}_{k}\left(\widetilde{p}_{k, n}^{0,(\mathrm{~S})}, \theta_{k, n}^{0}\right)} \geq q, \quad \forall k \in\{1, \ldots, K\} .
$$

Hence, one can simplify it as

$$
\begin{aligned}
f_{k}\left(q, \widetilde{p}_{k, n}^{0,(\mathrm{~S})}, \widetilde{p}_{k, n}^{0,(\mathrm{R})}, \theta_{k, n}^{0}\right)=\widetilde{r}_{k}\left(\widehat{p}_{k, n}^{0,(\mathrm{~S})}, \widetilde{p}_{k, n}^{0,(\mathrm{R})}, \theta_{k, n}^{0}\right) \\
-q \widetilde{p}_{k}\left(\widetilde{p}_{k, n}^{0,(\mathrm{~S})}, \theta_{k, n}^{0}\right) \geq 0, \quad \forall k \in\{1, \ldots, K\} .
\end{aligned}
$$

Accordingly,

$$
\begin{aligned}
f^{(1)}\left(q, \widetilde{p}_{k, n}^{0,(\mathrm{~S})}, \widetilde{p}_{k, n}^{0,(\mathrm{R})}, \theta_{k, n}^{0}\right) \\
\quad=\min _{1 \leq k \leq K} f_{k}\left(q, \widetilde{p}_{k, n}^{0,(\mathrm{~S})}, \widetilde{p}_{k, n}^{0,(\mathrm{R})}, \theta_{k, n}^{0}\right) \geq 0 .
\end{aligned}
$$

Since $f^{(1)}\left(q, \widetilde{p}_{k, n}^{0,(\mathrm{~S})}, \widetilde{p}_{k, n}^{0,(\mathrm{R})}, \theta_{k, n}^{0}\right) \geq 0$ at a feasible point $\left(\widetilde{p}_{k, n}^{0,(\mathrm{~S})}, \widetilde{p}_{k, n}^{0,(\mathrm{R})}, \theta_{k, n}^{0}\right)$, this implies

$$
f^{(2)}(q)=\max _{\left(\widetilde{\mathbf{P}}^{(\mathrm{S})}, \widetilde{\mathbf{P}}^{(\mathrm{R})}, \boldsymbol{\Theta}\right) \in \mathcal{A}} f^{(1)}\left(q, \widetilde{p}_{k, n}^{(\mathrm{S})}, \widetilde{p}_{k, n}^{(\mathrm{R})}, \theta_{k, n}\right) \geq 0 .
$$

-On the other hand, given that $f^{(2)}(q)=$ $\max _{\left.\widetilde{\mathbf{P}}^{(\mathrm{R})}, \boldsymbol{\Theta}\right) \in \mathcal{A}} f^{(1)}\left(q, \widetilde{p}_{k, n}^{\mathrm{S})}, \widetilde{p}_{k, n}^{(\mathrm{R})}, \theta_{k, n}\right) \geq 0$, then there $\left(\widetilde{\mathbf{P}}^{(\mathrm{S})}, \widetilde{\mathbf{P}}^{(\mathrm{R})}, \boldsymbol{\Theta}\right) \in \mathcal{A}$

exist some feasible point $\left(\widetilde{p}_{k, n}^{0,(\mathrm{~S})}, \widetilde{p}_{k, n}^{0,(\mathrm{R})}, \theta_{k, n}^{0}\right)$ such that

$$
\begin{aligned}
f^{(1)}\left(q, \widetilde{p}_{k, n}^{0,(\mathrm{~S})}, \widetilde{p}_{k, n}^{0,(\mathrm{R})}, \theta_{k, n}^{0}\right) \\
\quad=\min _{1 \leq k \leq K} f_{k}\left(q, \widetilde{p}_{k, n}^{0,(\mathrm{~S})}, \widetilde{p}_{k, n}^{0,(\mathrm{R})}, \theta_{k, n}^{0}\right) \geq 0,
\end{aligned}
$$

which can be further simplified as

$$
\begin{array}{lr}
\widetilde{r}_{k}\left(\widetilde{p}_{k, n}^{0,(\mathrm{~S})}, \widetilde{p}_{k, n}^{0,(\mathrm{R})}, \theta_{k, n}^{0}\right)-q \widetilde{p}_{k}\left(\widetilde{p}_{k, n}^{0,(\mathrm{~S})}, \theta_{k, n}^{0}\right) \geq 0, \\
& \forall k \in\{1, \ldots, K\}, \\
\frac{\widetilde{r}_{k}\left(\widetilde{p}_{k, n}^{0,(\mathrm{~S})}, \widetilde{p}_{k, n}^{0,(\mathrm{R})}, \theta_{k, n}^{0}\right)}{\widetilde{p}_{k}\left(\widetilde{p}_{k, n}^{0,(\mathrm{~S})}, \theta_{k, n}^{0}\right)} \geq q & \forall k \in\{1, \ldots, K\} .
\end{array}
$$

Since (55) holds for $\forall k \in\{1, \ldots, K\}$, this implies

$$
q^{*}\left(\widetilde{p}_{k, n}^{0,(\mathrm{~S})}, \widetilde{p}_{k, n}^{0,(\mathrm{R})}, \theta_{k, n}^{0}\right)=\min _{1 \leq k \leq K} \frac{\widetilde{r}_{k}\left(\widetilde{p}_{k, n}^{0,(\mathrm{~S})}, \widetilde{p}_{k, n}^{0,(\mathrm{R})}, \theta_{k, n}^{0}\right)}{\widetilde{p}_{k}\left(\widetilde{p}_{k, n}^{0,(\mathrm{~S})}, \theta_{k, n}^{0}\right)} \geq q .
$$

From the definition of $q^{*}$ in (16), (56) leads to

$$
\begin{aligned}
q^{*}=\max _{\left(\widetilde{\mathbf{P}}^{(\mathrm{S})}, \widetilde{\mathbf{P}}^{(\mathrm{R})}, \boldsymbol{\Theta}\right) \in \mathcal{A}} q^{*}\left(\widetilde{p}_{k, n}^{(\mathrm{S})}, \widetilde{p}_{k, n}^{(\mathrm{R})}, \theta_{k, n}\right) \\
\geq q^{*}\left(\widetilde{p}_{k, n}^{0,(\mathrm{~S})}, \widetilde{p}_{k, n}^{0,(\mathrm{R})}, \theta_{k, n}^{0}\right) \geq q .
\end{aligned}
$$

Finally, from (52) and (57) part (a) of Lemma 1 is proved. Similarly, one can prove part (b). Part (c) follows directly from parts (a) and (b). This concludes the proof of Lemma 1.

\section{APPENDIX D \\ PROOF OF THEOREM 1}

Since $q^{*} \leq \pi^{*}$ [29], we need to show that $q^{*} \geq \pi^{*}$ for finite values of $q^{*}$.

The function $\widetilde{p}_{k}\left(\widetilde{p}_{k, n}^{(\mathrm{S})}, \theta_{k, n}\right)$ is positive affine/affine bifunction and the function $\widetilde{r}_{k}\left(\widehat{p}_{k, n}^{(\mathrm{S})}, \widetilde{p}_{k, n}^{(\mathrm{R})}, \theta_{k, n}\right)$ is finite-valued convcave/convex bifunction on $\mathcal{A} \times \mathcal{B}$. Additionally, one can see that for finite $q^{*}$ the function $f_{k}\left(q^{*}, \widetilde{p}_{k, n}^{(\mathrm{S})}, \widetilde{p}_{k, n}^{(\mathrm{R})}, \theta_{k, n}\right)=$ $\widetilde{r}_{k}\left(\widetilde{p}_{k, n}^{(\mathrm{S})}, \widetilde{p}_{k, n}^{(\mathrm{R})}, \theta_{k, n}\right)-q^{*} \widetilde{p}_{k}\left(\widetilde{p}_{k, n}^{(\mathrm{S})}, \theta_{k, n}\right)$ is a concave/convex bifunction on $\mathcal{A} \times \mathcal{B}$ and is continuous on $\left(\widetilde{p}_{k, n}^{(\mathrm{S})}, \widetilde{p}_{k, n}^{(\mathrm{R})}, \theta_{k, n}\right) \in \mathcal{A}$ for every $k \in\{1, \ldots, K\}$. Thus, one can show that for nonfractional functions the following holds [29]

$$
f^{(2)}(q)=\min _{1 \leq k \leq K} \max _{\widetilde{\mathbf{P}}^{(\mathrm{S})}, \widetilde{\mathbf{P}}^{(\mathrm{R})}, \boldsymbol{\Theta}} f_{k}\left(q, \widetilde{p}_{k, n}^{(\mathrm{S})}, \widetilde{p}_{k, n}^{(\mathrm{R})}, \theta_{k, n}\right),
$$

which can be further expressed as

$$
f^{(2)}(q)=\max _{\widetilde{\mathbf{P}}^{(\mathrm{S})}, \widetilde{\mathbf{P}}^{(\mathrm{R})}, \boldsymbol{\Theta}} f_{k_{0}}\left(q, \widetilde{p}_{k_{0}, n}^{(\mathrm{S})}, \widetilde{p}_{k_{0}, n}^{(\mathrm{R})}, \theta_{k_{0}, n}\right),
$$

for some $k_{0} \in\{1, \ldots, K\}$. With the help of Lemma 1, one can write

$$
\begin{array}{r}
f^{(2)}\left(q^{*}\right)=\max _{\widetilde{\mathbf{P}}^{(\mathrm{S})}, \widetilde{\mathbf{P}}^{(\mathrm{R})}, \boldsymbol{\Theta}} \widetilde{r}_{k_{0}}\left(\widetilde{p}_{k_{0}, n}^{(\mathrm{S})}, \widetilde{p}_{k_{0}, n}^{\mathrm{R})}, \theta_{k_{0}, n}\right) \\
-q^{*} \widetilde{p}_{k_{0}}^{(\mathrm{S})}\left(\widetilde{p}_{k_{0}, n}^{(\mathrm{S})}, \theta_{k_{0}, n}\right)=0 .
\end{array}
$$

This consequently leads to

$$
\begin{array}{r}
\widetilde{r}_{k_{0}}\left(\widetilde{p}_{k_{0}, n}^{(\mathrm{S})}, \widetilde{p}_{k_{0}, n}^{(\mathrm{R})}, \theta_{k_{0}, n}\right)-q^{*} \widetilde{p}_{k_{0}}^{(\mathrm{S})}\left(\widetilde{p}_{k_{0}, n}^{(\mathrm{S})}, \theta_{k_{0}, n}\right) \leq 0, \\
\forall\left(\widetilde{p}_{k_{0}, n}^{(\mathrm{S})}, \widetilde{p}_{k_{0}, n}^{(\mathrm{R})}, \theta_{k_{0}, n}\right) \in \mathcal{A} .
\end{array}
$$

Since $\widetilde{p}_{k_{0}}^{(\mathrm{S})}\left(\widetilde{p}_{k_{0}, n}^{\mathrm{S})}, \theta_{k_{0}, n}\right)>0, \forall\left(\widetilde{p}_{k, n}^{(\mathrm{S})}, \widetilde{p}_{k, n}^{(\mathrm{R})}, \theta_{k, n}\right) \in \mathcal{A}$, we 
obtain

$$
\frac{\widetilde{r}_{k_{0}}\left(\widetilde{p}_{k_{0}, n}^{(\mathrm{S})}, \widetilde{p}_{k_{0}, n}^{(\mathrm{R})}, \theta_{k_{0}, n}\right)}{\widetilde{p}_{k_{0}}^{(\mathrm{S})}\left(\widetilde{p}_{k_{0}, n}^{(\mathrm{S})}, \theta_{k_{0}, n}\right)} \leq q^{*}, \quad \forall\left(\widetilde{p}_{k_{0}, n}^{(\mathrm{S})}, \widetilde{p}_{k_{0}, n}^{(\mathrm{R})}, \theta_{k_{0}, n}\right) \in \mathcal{A} .
$$

Sine (62) holds for $\forall\left(\widetilde{p}_{k_{0}, n}^{(\mathrm{S})}, \widetilde{p}_{k_{0}, n}^{(\mathrm{R})}, \theta_{k_{0}, n}\right) \in \mathcal{A}$, it follows that

$$
\max _{\widetilde{\mathbf{P}}^{(\mathrm{S})}, \widetilde{\mathbf{P}}^{(\mathrm{R})}, \Theta} \frac{\widetilde{r}_{k_{0}}\left(\widetilde{p}_{k_{0}, n}^{(\mathrm{S})}, \widetilde{p}_{k_{0}, n}^{(\mathrm{R})}, \theta_{k_{0}, n}\right)}{\widetilde{p}_{k_{0}}^{(\mathrm{S})}\left(\widetilde{p}_{k_{0}, n}^{(\mathrm{S})}, \theta_{k_{0}, n}\right)} \leq q^{*} .
$$

As (63) is valid for some $k_{0} \in\{1, \ldots, K\}$, this implies

$$
\begin{aligned}
\pi^{*}=\min _{1 \leq k \leq K} \max _{\widetilde{\mathbf{P}}^{(\mathrm{S})}, \widetilde{\mathbf{P}}^{(\mathrm{R})}, \Theta} \frac{\widetilde{r}_{k}\left(\widetilde{p}_{k, n}^{(\mathrm{S})}, \widetilde{p}_{k, n}^{(\mathrm{R})}, \theta_{k, n}\right)}{\widetilde{p}_{k}\left(\widetilde{p}_{k, n}^{(\mathrm{S})}, \theta_{k, n}\right)} \\
\leq \max _{\widetilde{\mathbf{P}}^{(\mathrm{S})}, \widetilde{\mathbf{P}}^{(\mathrm{R})}, \Theta} \frac{\widetilde{r}_{k_{0}}\left(\widetilde{p}_{k_{0}, n}^{(\mathrm{S})}, \widetilde{p}_{k_{0}, n}^{(\mathrm{R})}, \theta_{k_{0}, n}\right)}{\widetilde{p}_{k_{0}}^{(\mathrm{S})}\left(\widetilde{p}_{k_{0}, n}^{(\mathrm{S})}, \theta_{k_{0}, n}\right)} \leq q^{*} .
\end{aligned}
$$

As $q^{*} \leq \pi^{*}$ [29] and $q^{*} \geq \pi^{*}$ according to (64), hence it follows that $\pi^{*}=q^{*}$ and strong duality holds.

\section{REFERENCES}

[1] Ericsson, "Sustainable energy use in mobile communications," Aug. 2007.

[2] R. Bolla, R. Bruschi, F. Davoli, and F. Cucchietti, "Energy efficiency in the future internet: a survey of existing approaches and trends in energy-aware fixed network infrastructures," IEEE Commun. Surveys Tuts., vol. 13, no. 2, pp. 223-244, Second Quarter 2011.

[3] C. Han, T. Harrold, S. Armour, I. Krikidis, S. Videv, P. M. Grant, H. Haas, J. S. Thompson, I. Ku, C.-X. Wang et al., "Green radio: radio techniques to enable energy-efficient wireless networks," IEEE Commun. Mag., vol. 49, no. 6, pp. 46-54, May 2011.

[4] M. Dohler and Y. Li, Cooperative Communications: Hardware, Channel \& PHY. John Wiley \& Sons, 2010.

[5] E. Hossain, D. I. Kim, and V. K. Bhargava, Cooperative cellular wireless networks. Cambridge University Press, 2011.

[6] J. Laneman, D. Tse, and G. Wornell, "Cooperative diversity in wireless networks: Efficient protocols and outage behavior," IEEE Trans. Inf. Theory, vol. 50, no. 12, pp. 3062-3080, Dec. 2004.

[7] K. Fazel and S. Kaiser, Multi-carrier and Spread Spectrum Systems: from OFDM and MC-CDMA to LTE and WiMAX. John Wiley \& Sons Inc, 2008.

[8] C. Y. Wong, R. S. Cheng, K. B. Lataief, and R. D. Murch, "Multiuser OFDM with adaptive subcarrier, bit, and power allocation," IEEE J. Sel. Areas Commun., vol. 17, no. 10, pp. 1747-1758, Oct. 1999.

[9] M. Salem, A. Adinoyi, M. Rahman, H. Yanikomeroglu, D. Falconer, Y.-D. Kim, E. Kim, and Y.-C. Cheong, "An overview of radio resource management in relay-enhanced OFDMA-based networks," IEEE Commun. Surveys Tuts., vol. 12, no. 3, pp. 422-438, First Quarter 2010.

[10] M. S. Alam, J. W. Mark, and X. S. Shen, "Relay selection and resource allocation for multi-user cooperative OFDMA networks," IEEE Trans. Wireless Commun., vol. 12, no. 5, pp. 2193-2205, May 2013.

[11] K. Jitvanichphaibool, R. Zhang, and Y.-C. Liang, "Optimal resource allocation for two-way relay-assisted OFDMA," IEEE Trans. Veh. Technol., vol. 58, no. 7, pp. 3311-3321, Sep. 2009.

[12] J. Joung and S. Sun, "Power efficient resource allocation for downlink OFDMA relay cellular networks," IEEE Trans. Signal Process., vol. 60, no. 5, pp. 2447-2459, May 2012.

[13] Y. Chen, X. Fang, and Y. Zhao, "Energy-efficient adaptive power allocation in orthogonal frequency division multiplexing-based amplifyand-forward relay link," IET Communications, vol. 7, no. 15, pp. 16761687, Jul. 2013.

[14] C. Sun, Y. Cen, and C. Yang, "Energy efficient OFDM relay systems," IEEE Trans. Commun., vol. 61, no. 5, pp. 1797-1809, May 2013.

[15] R. Devarajan, S. Jha, U. Phuyal, and V. Bhargava, "Energy-aware resource allocation for cooperative cellular network using multi-objective optimization approach," IEEE Trans. Wireless Commun., vol. 11, no. 5, pp. 1797-1807, May 2012.

[16] C. Y. Ho and C.-Y. Huang, "Energy efficient subcarrier-power allocation and relay selection scheme for OFDMA-based cooperative relay networks," in in Proc. Int. Conf. on Commun. (ICC), 2011, pp. 1-6.
[17] K. T. K. Cheung, S. Yang, and L. Hanzo, "Achieving maximum energy-efficiency in multi-relay OFDMA cellular networks: A fractional programming approach," IEEE Trans. Commun., vol. 61, no. 7, pp. 2746-2757, Jul. 2013.

[18] R. Arab Loodaricheh, S. Mallick, and V. Bhargava, "Energy efficient resource allocation for OFDMA cellular networks with user cooperation and QoS provisioning," IEEE Trans. Wireless Commun., vol. 13, no. 11, pp. 6132-6146, Nov. 2014.

[19] C. Xiong, G. Y. Li, S. Zhang, Y. Chen, and S. Xu, "Energy-efficient resource allocation in OFDMA networks," IEEE Trans. Commun., vol. 60 , no. 12 , pp. 3767-3778, Dec. 2012.

[20] M. O. Hasna and M.-S. Alouini, "End-to-end performance of transmission systems with relays over Rayleigh-fading channels," IEEE Trans. Wireless Commun., vol. 2, no. 6, pp. 1126-1131, Nov. 2003.

[21] Y. Shen, G. Feng, B. Yang, and X. Guan, "Fair resource allocation and admission control in wireless multiuser amplify-and-forward relay networks," IEEE Trans. Veh. Technol., vol. 61, no. 3, pp. 1383-1397, Mar. 2012.

[22] J. Tang and X. Zhang, "Cross-layer resource allocation over wireless relay networks for quality of service provisioning," IEEE J. Sel. Areas Commun., vol. 25, no. 4, pp. 645-656, May 2007.

[23] C. A. Floudas, Nonlinear and Mixed-Integer Optimization: Fundamentals and Applications. Oxford University Press, USA, 1995.

[24] W. Yu and R. Lui, "Dual methods for nonconvex spectrum optimization of multicarrier systems," IEEE Trans. Commun., vol. 54, no. 7, pp. 13101322, Jul. 2006.

[25] Z.-Q. Luo and S. Zhang, "Dynamic spectrum management: Complexity and duality," IEEE J. Sel. Topics Signal Process., vol. 2, no. 1, pp. 5773, Feb. 2008

[26] K. Seong, M. Mohseni, and J. M. Cioffi, "Optimal resource allocation for OFDMA downlink systems," in in Proc. IEEE International Symposium on Information Theory, Jul. 2006, pp. 1394-1398.

[27] S. Schaible, "Fractional programming," in Encyclopedia of Operations Research and Management Science. Springer, 2013, pp. 605-608.

[28] S. Boyd and L. Vandenberghe, Convex Optimization. Cambridge University Press, 2004.

[29] J. Frenk and G. Kassay, "Minimax results and finite-dimensional separation," Journal of Optimization Theory and Applications, vol. 113, no. 2, pp. 409-421, 2002.

[30] J. B. G. Frenk, G. Kassay, and J. Kolumbán, "On equivalent results in minimax theory," European Journal of Operational Research, vol. 157, no. 1, pp. 46-58, 2004.

[31] W. Dinkelbach, "On nonlinear fractional programming," Management Science, vol. 13, no. 7, pp. 492-498, Mar. 1967.

[32] W. P. Siriwongpairat, A. K. Sadek, and K. R. Liu, "Cooperative communications protocol for multiuser OFDM networks," IEEE Trans. Wireless Commun., vol. 7, no. 7, pp. 2430-2435, Jul. 2008.

[33] R. Jain, D.-M. Chiu, and W. Hawe, "A quantitative measure of fairness and discrimination for resource allocation in shared computer systems," Digital Equipment Corporation, DEC-TR-301, Tech. Rep., 1982.

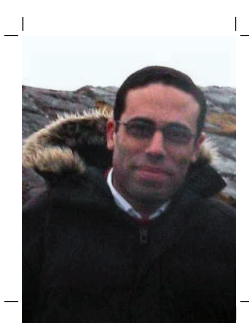

Ebrahim Bedeer (S'10, M'14) received the B.Sc. and M.Sc. degrees from Tanta University, Tanta, Egypt, in 2002 and 2008, respectively and the Ph.D. degree from Memorial University, St. Johns, NL, Canada in 2014, all in Electrical Engineering. Currently, he is a postdoctoral fellow at University of British Columbia (UBC), Kelowna, BC, Canada. His current research interests are in the area of wireless communications, with a focus on dynamic resource allocation, green communications, cooperative communication, and cognitive radio networks.

Dr. Bedeer has served on the Technical Program Committees of numerous major international communication conferences, such as IEEE GLOBECOM, IEE ICC, IEEE VTC, and CROWNCOM. 


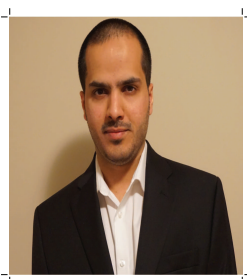

Abdulaziz Alorainy (S'14) received the B.Sc. degree in electrical engineering from King Fahd University of Petroleum and Minerals (KFUPM), Dhahran, Saudi Arabia, in 2010 and the M.A.Sc. degree from King Abdullah University of Science and Technology (KAUST), Thuwal, Saudi Arabia, in 2012. He is currently working towards the Ph.D. degree at the University of British Columbia (UBC), Kelowna, BC, Canada. Since 2010, he has been working as a research assistant at King Abdulaziz City for Science and Technology (KACST), Riyadh, Saudi Arabia. His research interests include green communications, crosslayer analysis and communication quality of service.

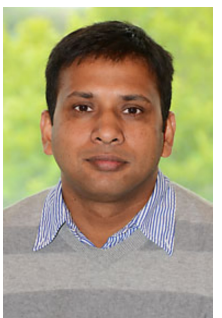

Jahangir Hossain (S'04, M'08) received the B.Sc. degree in electrical and electronics engineering from Bangladesh University of Engineering and Technology (BUET), Dhaka, Bangladesh; the M.A.Sc. degree from the University of Victoria, Victoria, BC, Canada, and the Ph.D. degree from the University of British Columbia (UBC), Vancouver, BC, Canada.

He served as a Lecturer at BUET. He was a Research Fellow with McGill University, Montreal, QC, Canada; the National Institute of Scientific Research, Quebec, QC, Canada; and the Institute for Telecommunications Research, University of South Australia, Mawson Lakes, Australia. His industrial experiences include a Senior Systems Engineer position with Redline Communications, Markham, ON, Canada, and a Research Intern position with Communication Technology Lab, Intel, Inc., Hillsboro, OR, USA. He is currently working as an Assistant Professor in the School of Engineering, UBC Okanagan campus, Kelowna, BC, Canada. His research interests include designing spectrally and power-efficient modulation schemes, quality of service issues and resource allocation in wireless networks. Dr. Hossain regularly serves as a member of the Technical Program Committee of the IEEE International Conference on Communications (ICC). He was an Editor for the IEEE Transactions on Wireless Communications. He received the Natural Sciences and Engineering Research Council of Canada Postdoctoral Fellowship.

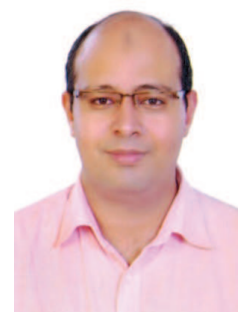

Osama Amin (S'07, M'11, SM'15) received B.Sc. degree in Electrical and Electronics Engineering from Aswan University, Aswan, Egypt, in 2000, M.Sc. degree in Electrical Engineering from Assiut University, Assiut, Egypt in 2004 and Ph.D. degree in Electrical and Computer Engineering, University of Waterloo, Canada in 2010. In June 2012, he joined Assiut University as an Assistant Professor in the Electrical and Electronics Engineering department. Currently, he is with King Abdullah University of Science and Technology (KAUST), Thuwal, Makkah, Kingdom of Saudi Arabia. His general research interests lie in communications systems and signal processing for communications with special emphasis on wireless applications. Specific research areas include green communications, cognitive radio, cooperative communications, and channel estimation. He served as a technical program committee (TPC) member for IEEE VTC, CROWNCOM, PIMRC, and ISSPIT conferences. $\mathrm{He}$ is a co-organizer and a co-chair of the Next Generation Green ICT and 5G Networking (GreeNets) 2015 in the IEEE International Conference on Ubiquitous Wireless Broadband (ICUWB) in Montreal, Canada.

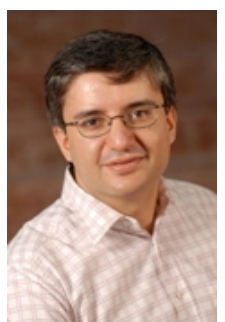

Mohamed-Slim Alouini (S'94, M'98, SM'03, F'09) was born in Tunis, Tunisia. He received the Ph.D. degree in Electrical Engineering from the California Institute of Technology (Caltech), Pasadena, CA, USA, in 1998. He served as a faculty member in the University of Minnesota, Minneapolis, MN, USA, then in the Texas A\&M University at Qatar, Education City, Doha, Qatar before joining King Abdullah University of Science and Technology (KAUST), Thuwal, Makkah Province, Saudi Arabia as a Professor of Electrical Engineering in 2009. His current research interests include the modeling, design, and performance analysis of wireless communication systems. 